\title{
Tanycytic networks mediate energy balance by feeding lactate to glucose-insensitive POMC neurons
}

\author{
Tori Lhomme, ${ }^{1}$ Jerome Clasadonte, ${ }^{1}$ Monica Imbernon, ${ }^{1}$ Daniela Fernandois, ${ }^{1}$ Florent Sauve, ${ }^{1}$ Emilie Caron, ${ }^{1}$ Natalia da Silva Lima, ${ }^{2,3}$ \\ Violeta Heras, ${ }^{2,3}$ Ines Martinez-Corral, ${ }^{1}$ Helge Mueller-Fielitz, ${ }^{4}$ Sowmyalakshmi Rasika, ${ }^{1}$ Markus Schwaninger, ${ }^{4}$ \\ Ruben Nogueiras, ${ }^{2,3}$ and Vincent Prevot ${ }^{1}$ \\ 'University Lille, Inserm, CHU Lille, Laboratory of Development and Plasticity of the Neuroendocrine Brain, Lille Neuroscience and Cognition, UMR-S 1172, European Genomic Institute of Diabetes (EGID) and \\ Development of Innovative Strategies for a Transdisciplinary Approach to Alzheimer's Disease (DISTALZ), Lille, France. ²IMUS, Universidade de Santiago de Compostela-Instituto de Investigación Sanitaria, \\ Santiago de Compostela, Spain. ${ }^{3}$ CIBER Fisiopatología de la Obesidad y Nutrición (CIBERobn), Santiago de Compostela, Spain. ${ }^{4}$ Institute for Experimental and Clinical Pharmacology and Toxicology, \\ University of Lübeck, Lübeck, Germany.
}

\begin{abstract}
Hypothalamic glucose sensing enables an organism to match energy expenditure and food intake to circulating levels of glucose, the main energy source of the brain. Here, we established that tanycytes of the arcuate nucleus of the hypothalamus, specialized glia that line the wall of the third ventricle, convert brain glucose supplies into lactate that they transmit through monocarboxylate transporters to arcuate proopiomelanocortin neurons, which integrate this signal to drive their activity and to adapt the metabolic response to meet physiological demands. Furthermore, this transmission required the formation of extensive connexin-43 gap junction-mediated metabolic networks by arcuate tanycytes. Selective suppression of either tanycytic monocarboxylate transporters or gap junctions resulted in altered feeding behavior and energy metabolism.

Tanycytic intercellular communication and lactate production are thus integral to the mechanism by which hypothalamic neurons that regulate energy and glucose homeostasis efficiently perceive alterations in systemic glucose levels as a function of the physiological state of the organism.
\end{abstract}

\section{Introduction}

Glucose plays a dual role in the brain: it is both an essential fuel that sustains brain activity and an indicator of peripheral energy status. Circulating glucose levels fluctuate as a function of food intake or energy expenditure. However, the entrance of glucose into the brain is regulated by specific transport systems $(1,2)$ that ensure that extracellular glucose concentrations in the brain $(\sim 2 \mathrm{mM})$, while significantly lower than those in the blood $(\sim 5 \mathrm{mM})$, remain constant to fulfill the energy needs of brain cells, regardless of the physiological state of the individual (3). How then do brain networks whose function it is to maintain energy homeostasis by modulating metabolic pathways detect changes in peripheral energy status? The arcuate nucleus of the hypothalamus (ARH), which flanks the third ventricle $(3 \mathrm{~V})$ on both sides, contains discrete populations of neurons that are critically involved in the regulation of energy homeostasis $(4,5)$. Among them, the pro-opiomelanocortin (POMC) neurons $(6,7)$, which are electrically excited by glucose, are

\section{Related Commentary: https://doi.org/10.1172/JCl153279}

Authorship note: JC, MI, and DF contributed equally to this work. MS, RN, and VP jointly supervised this work.

Conflict of interest: The authors have declared that no conflict of interest exists. Copyright: () 2021, American Society for Clinical Investigation.

Submitted: May 21, 2020; Accepted: July 28, 2021; Published: September 15, 2021

Reference information: / Clin Invest. 2021;131(18):e140521.

https://doi.org/10.1172/JCl140521. well placed to adjust food intake and energy expenditure in response to changes in circulating glucose levels (8). However, whether POMC neurons are themselves capable of sensing glucose remains controversial $(9,10)$. Instead, it seems logical for this role to be assumed by a different class of cells that is in touch, on the one hand, with both the blood and the cerebrospinal fluid (CSF), whose glucose concentrations vary in proportion to blood concentrations $(11,12)$ and, on the other hand, with POMC neurons, and that can therefore transmit changes in glucose levels in these 2 compartments or translate them into a signal that the neurons can perceive.

Here, we tested the hypothesis that POMC neuronal activity is not directly controlled by glucose but by its metabolite lactate (13), and we asked whether tanycytes, peculiar glial cells constituting a CSF-brain barrier along the wall of the $3 \mathrm{~V}$ that replaces the traditional blood-brain barrier $(14,15)$, act as the source of the metabolic signal required by POMC neurons for hypothalamic glucose sensing and the control of energy homeostasis.

\section{Results}

POMC neurons use lactate and not glucose as an energy substrate. Many glucose-sensing neurons respond to or integrate signals from glucose metabolites, especially lactate (10). To determine whether POMC neurons also use lactate as an energy substrate, we used whole-cell current-clamp recordings in acute brain slices from tdTomato $^{P O M C}$ mice (Figure 1A). In the presence of physiological levels of glucose $(2.5 \mathrm{mM})$, POMC neurons exhibited tonic firing. However, the bath application of a selective inhib- 
A

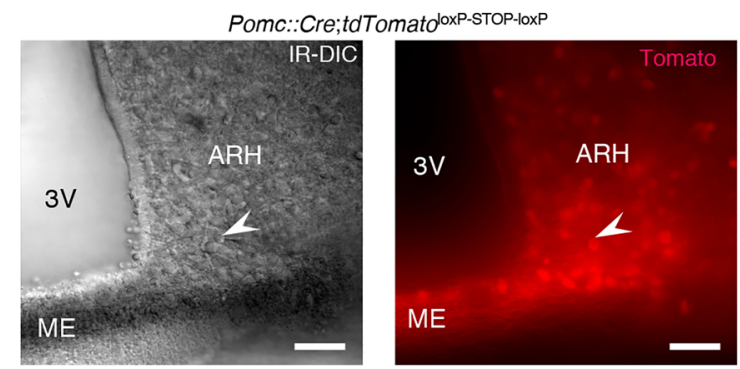

B
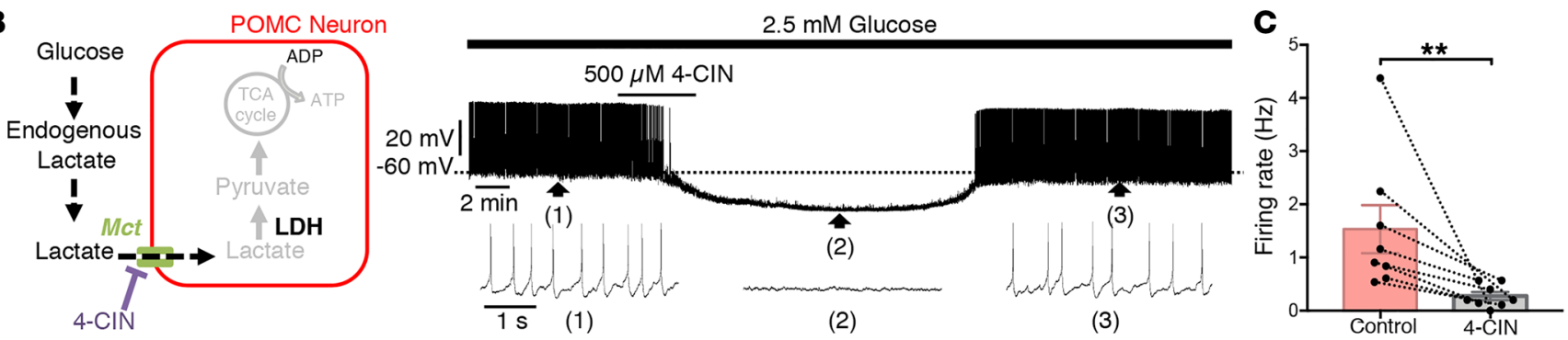

D

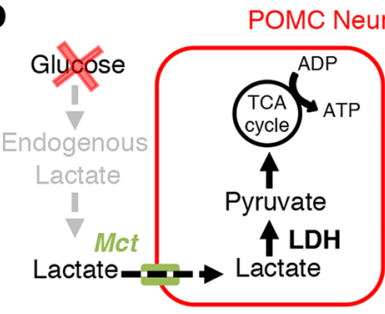

$2.5 \mathrm{mM}$ Glucose

(3)
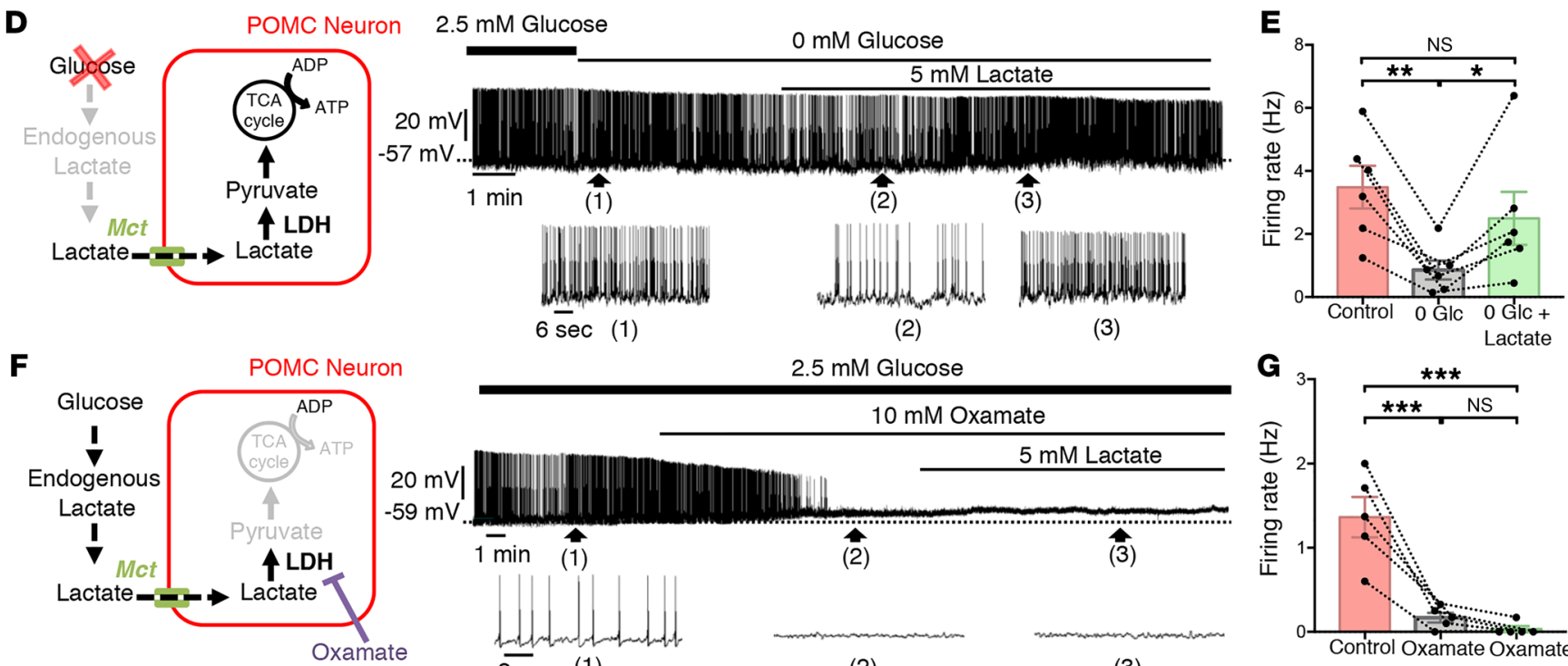

$0 \mathrm{mM}$ Glucose

H

Pyruvate
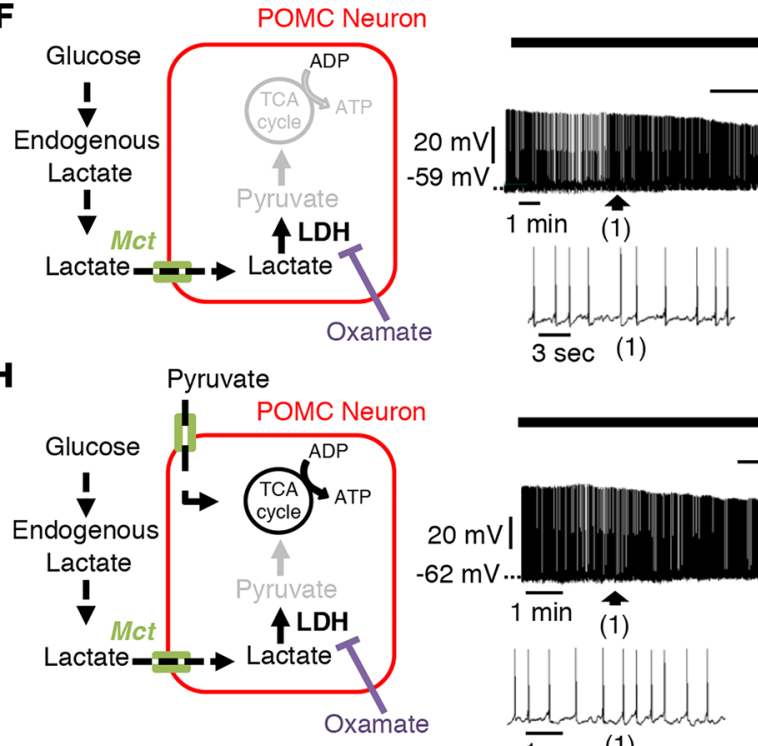

$2.5 \mathrm{mM}$ Glucose

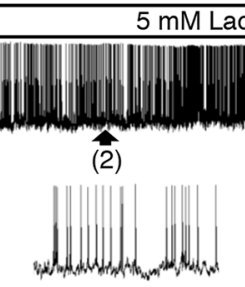

(2)

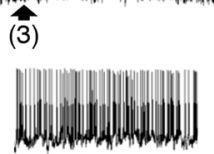

(3)

(3)
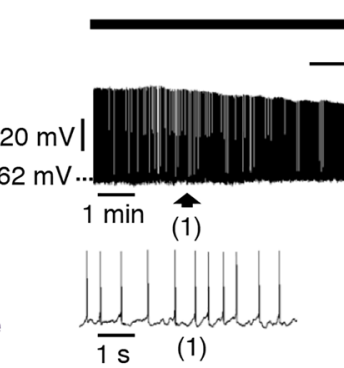

(2)

(3)

$2.5 \mathrm{mM}$ Glucose

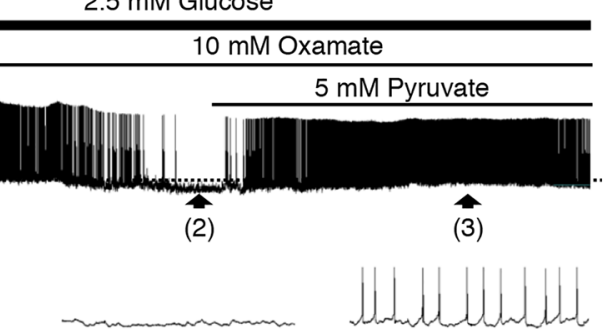

(2)

(3)

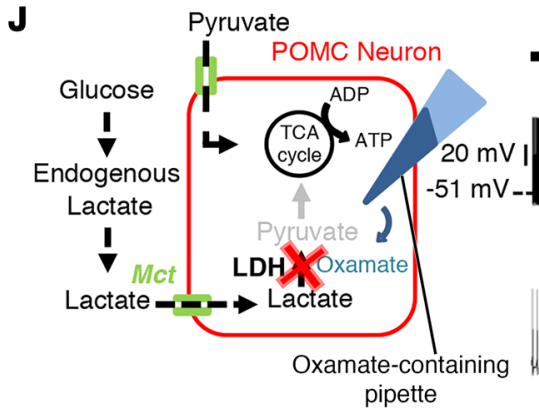

$2.5 \mathrm{mM}$ Glucose (Bath) + $10 \mathrm{mM}$ Oxamate (POMC neuron)

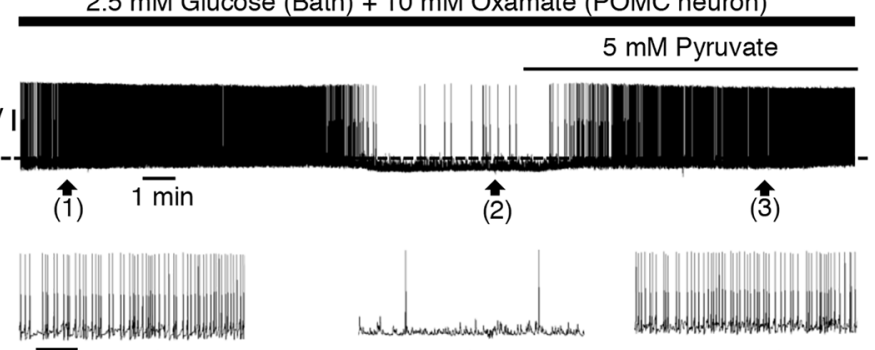

(1)

(3)

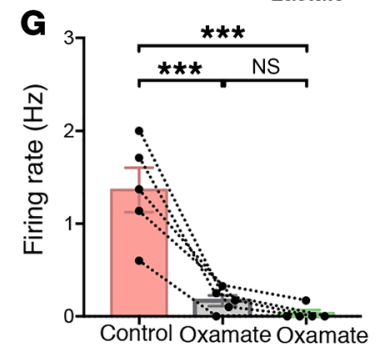

+ Lactate
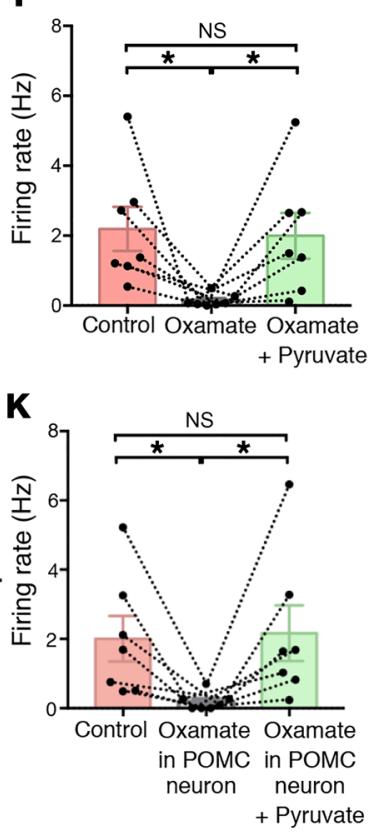
Figure 1. Endogenous production of lactate sustains POMC neuronal activity. (A) Infrared differential interference contrast (IR-DIC) image (left) of the ARH at the level of the ME showing a patch-clamp electrode placed onto the cell body of a POMC neuron identified via fluorescence (right) in a tdTomato ${ }^{\text {POMC }}$ mouse. Scale bars: $50 \mu \mathrm{m}$. (B) Schematic model representing the modulation of POMC neuronal activity by lactate. Whole-cell current-clamp recording of a POMC neuron performed in ACSF containing a physiological concentration of glucose, showing that bath application of 4-CIN, an inhibitor of MCTs, blunted its spontaneous neuronal activity. Bottom traces show expansions of the recording at the indicated time points 1,2 , and 3 . (C) Firing rate of POMC neurons before and after the bath application of 4-CIN. ( $D$ and $\mathbf{E}$ ) A switch in ACSF glucose concentrations from 2.5 to $0 \mathrm{mM}$ decreased the spontaneous firing rate of POMC neurons. In the same neuron, lactate reversed the glucose deprivation effect. (F) Inhibition of LDH with oxamate inhibited spontaneous POMC neuronal activity in ACSF with $2.5 \mathrm{mM}$ glucose. Lactate, the substrate of $L D H$, did not reverse the inhibitory effect of oxamate on the same neuron. (C) Lack of compensation by lactate of the inhibitory effect of oxamate on the firing rate of POMC neurons. (H and I) Bath application of the LDH inhibitor oxamate inhibited the activity of POMC neurons in ACSF with $2.5 \mathrm{mM}$ glucose. In the same neuron, pyruvate, the metabolite of LDH, reversed the inhibitory effect of oxamate. ( and $\mathbf{K}$ ) Intracellular application of oxamate in POMC neurons inhibited their activity in ACSF with $2.5 \mathrm{mM}$ glucose. In the same neuron, the bath application of pyruvate reversed the inhibitory effect of oxamate. ${ }^{*} P$ $<0.05,{ }^{* *} P<0.01$, and ${ }^{* *} P<0.001$, by Wilcoxon matched-pairs test (C) and repeated-measures 1-way ANOVA with Tukey's post hoc test (E, G, I, K).

itor of the monocarboxylate transporters (MCTs), $\alpha$-cyano-4hydroxycinnamate (4-CIN, $500 \mu \mathrm{M})$, to block lactate influx into cells (16) completely inhibited this tonic firing $(P=0.0078$, Wilcoxon matched-pairs test, $n=8$ cells, $n=5$ mice; Figure 1 , B and $\mathrm{C})$, indicating that lactate, and not glucose, was fueling POMC neuronal activity. We next induced glucose deprivation by switching from $2.5 \mathrm{mM}$ to $0 \mathrm{mM}$ glucose in artificial cerebrospinal fluid (ACSF), which caused a marked decrease in POMC neuron firing $(P=0.0015,1$-way, repeated-measures ANOVA followed by Tukey's post hoc test, $n=6$ cells, $n=4$ mice). The bath application of $5 \mathrm{mM}$ lactate (equicaloric to $2.5 \mathrm{mM}$ glucose; ref. 16 ) restored the spontaneous firing frequency of glucose-deprived POMC neurons $(P=0.0280, n=6$ cells, $n=4$ mice; Figure $1, \mathrm{D}$ and $\mathrm{E})$. This rescue effect was in turn abolished by the bath application of $10 \mathrm{mM}$ oxamate $(P=0.0277,1$-way repeated-measures ANOVA followed by Tukey's post hoc test, $n=7$ cells, $n=5$ mice; Figure 1 , F-I), which inhibits lactate dehydrogenase (LDH), an enzyme responsible for the conversion of lactate to pyruvate, the metabolite that principally fuels neurons through its entry into the tricarboxylic acid cycle (17). In accordance with this sequence of events, although the addition of lactate did not compensate for the loss of neuronal activity induced by oxamate $(P=0.7811,1$-way, repeated-measures ANOVA followed by Tukey's post hoc test, $n=5$ cells, $n=4$ mice; Figure $1, \mathrm{~F}$ and $\mathrm{G})$, the bath application of pyruvate completely reversed it $(5 \mathrm{mM})(P=0.046,1$-way, repeated-measures ANOVA followed by Tukey's post hoc test, $n=7$ cells, $n=5$ mice; Figure $1, \mathrm{H}$ and I). To ascertain that changes in POMC neuronal activity were not due to an indirect action of oxamate on neighboring neurons, oxamate was directly delivered into the cytoplasm of the recorded cell via the patch pipette (Figure 1J). This cell-restricted LDH inhibition also blunted neuronal activity $(P=0.0236,1$-way repeated-measures ANOVA followed by Tukey's post hoc test, $n=7$ cells, $n=5$ mice $)$ in a pyruvate-reversible manner $(P=0.0145, n=7$ cells, $n$ $=5$ mice; Figure $1, \mathrm{~J}$ and $\mathrm{K})$. Altogether, these results suggest that rather than glucose, lactate produced locally from glucose in living brain slices is taken up by POMC neurons via MCTs and sustains their neuronal activity through its conversion to pyruvate.

Tanycytes shuttle lactate to POMC neurons. ARH tanycytes, thanks to the position of their cell bodies lining the walls of the $3 \mathrm{~V}$ and end-feet that contact vessels of the blood-brain barrier (14), are in contact with both blood-borne and CSF-borne glucose and also express glucose-sensing machinery reminiscent of that found in pancreatic $\beta$ cells (18-20). In addition, tanycytes express functional MCTs $(21,22)$. To determine whether tanycytes, rather than the astrocytic lactate shuttle that feeds neuronal activity in other brain areas $(23,24)$, could be the source of the endogenous lactate required by POMC neurons, we first performed experiments in primary cultures of tanycytes, which we have extensively characterized previously $(25,26)$. Immunofluorescence experiments showed that cultured tanycytes expressed the lactate transporters MCT1 and MCT4 (Figure 2, A and B). In addition, the spontaneous lactate release seen from tanycytes cultured in glucose-containing, serum-free defined medium (Figure 2C) was blunted by the addition to the culture medium of the non-metabolizable glucose ana$\log$ 2-deoxyglucose (2-DG, $50 \mathrm{mM})(P=0.0016$, ordinary 1-way ANOVA followed by Tukey's post hoc test, $n=5$; Figure $2 \mathrm{C}$ ), which also blunted lactate production within the cells $(P=0.0001$, ordinary 1-way ANOVA followed by Tukey's post hoc test, $n=5$; Figure 2D). Since MCTs are responsible for lactate exit from cells, inhibition of tanycytic MCTs by treating the cultures for 30 minutes with $500 \mu \mathrm{M} 4-\mathrm{CIN}$ abrogated the ability of tanycytes to release lactate $(P=0.012, n=6$; Figure 2C) and caused an accumulation of lactate in their cytoplasm $(P=0.0001, n=6$; Figure $2 D)$. These in vitro results indicate that tanycytes could indeed produce and release lactate through their uptake and metabolization of glucose.

Next, to test the possibility that lactate released by tanycytes fuels POMC neurons in the ARH, we asked whether the delivery of lactate into ARH tanycytes could rescue activity in POMC neurons during glucose deprivation in living brain slices. We dialyzed a single tanycyte with $5 \mathrm{mM}$ lactate while performing a whole-cell recording from a distant POMC neuron (50-200 $\mu \mathrm{m}$ apart; Figure 2, E-J, and Supplemental Figure 1, A-C; supplemental material available online with this article; https://doi.org/10.1172/ JCI140521DS1). When the tanycyte was dialyzed with lactate, the reduction in action potential firing seen with glucose deprivation in Figure 1D no longer occurred ( $P=0.9974,1$-way, repeated-measures ANOVA followed by Tukey's post hoc test, $n=6$ cells, $n=5$ mice; Figure 2, $\mathrm{H}$ and I); this was true of all the POMC neurons that were patched ( $n=9$; including some not shown), even when the neurons were at a considerable distance from the patched tanycyte and not in the path of the tanycytic process (Figure 2J). This tanycyte-mediated rescue of POMC neuronal firing was reversed with the loss of the tanycytic patch that sometimes occurred during the course of the experiment (Supplemental Figure 1D), but could be counteracted by bath application of $5 \mathrm{mM}$ lactate (Supplemental Figure 1D) as shown in Figure 1D. We next explored whether selective blocking of lactate production in the patched tanycytes would have an impact on the activity of the POMC neurons with which they are connected, regardless of the presence of physiological levels of glucose (2.5 mM; Figure $2 \mathrm{~K})$. When a single tanycyte was dialyzed with the LDH inhibitor oxamate to block the conver- 
A

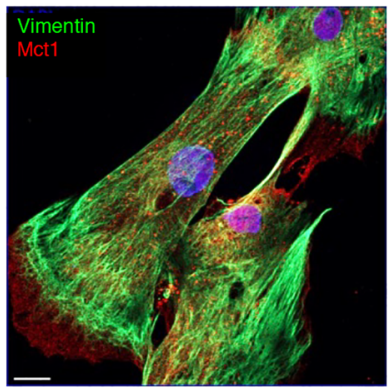

B

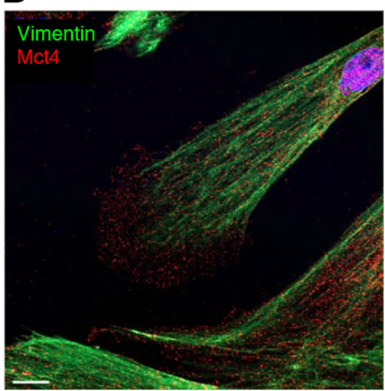

E
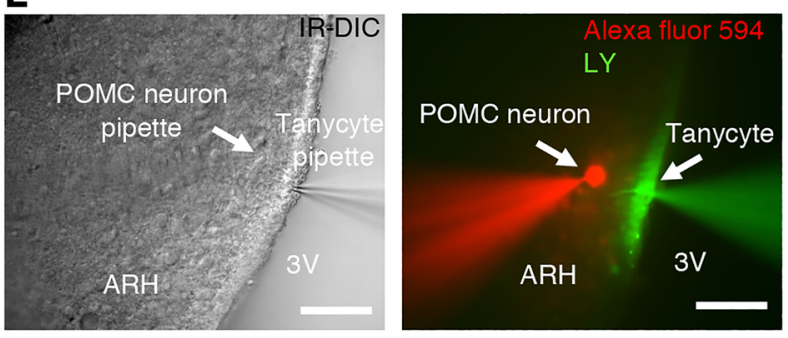

G

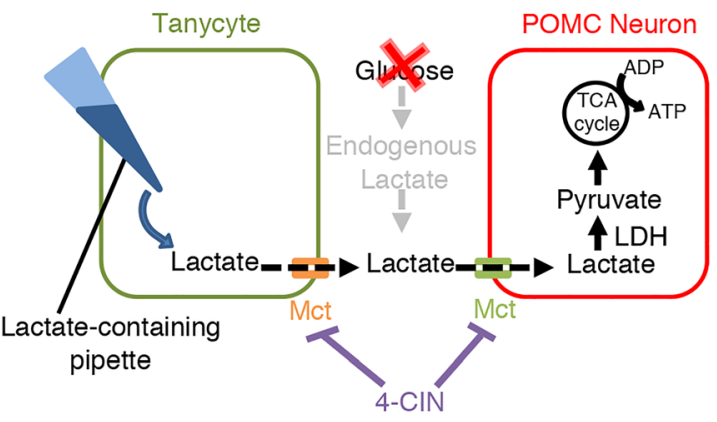

H

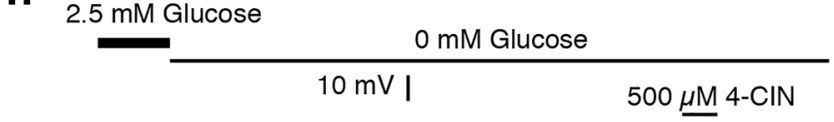

$76 \mathrm{mV}$ Tanycyte $+5 \mathrm{mM}$ Lactate

POMC Neuron

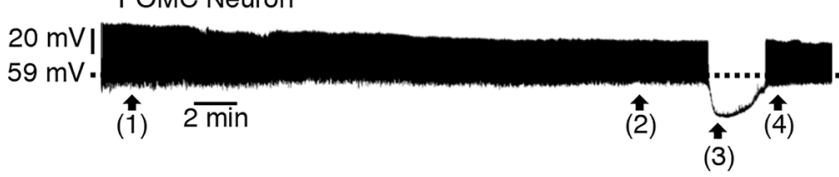

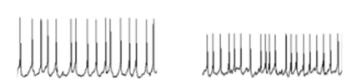
$\overline{2 s}(1)$

(2)

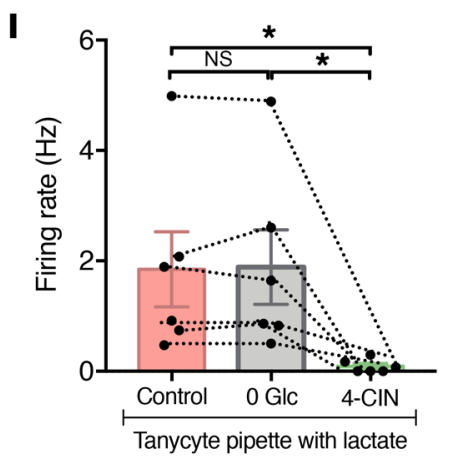

(3)

(4)

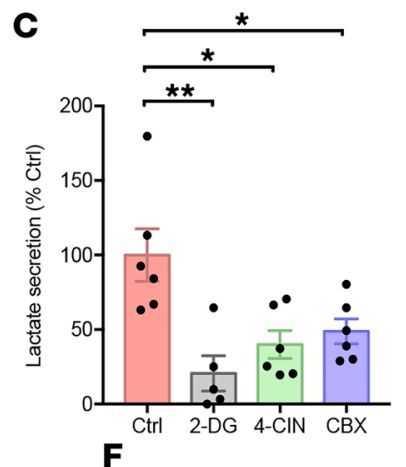

$\mathbf{F}$

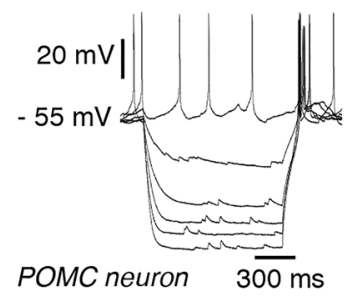

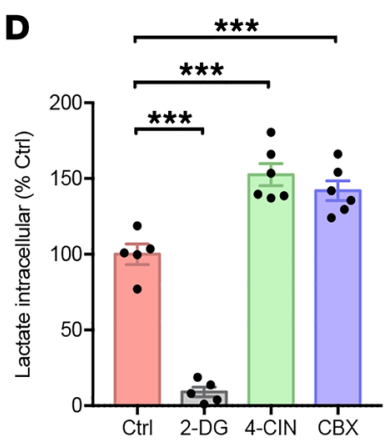

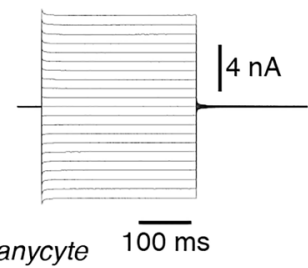

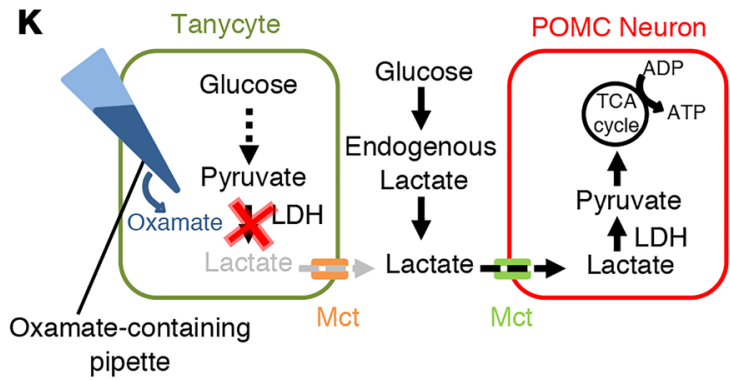

$\mathbf{L}$

$2.5 \mathrm{mM}$ Glucose $-78 \mathrm{mV}$

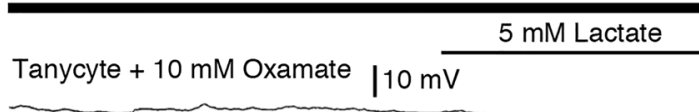

POMC neuron

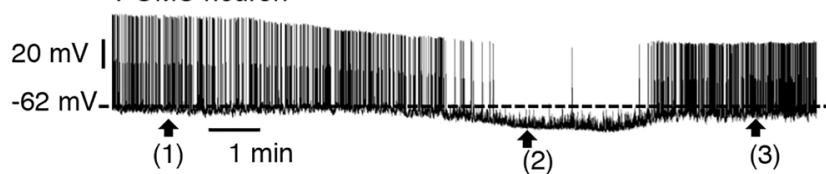

(1) 1 min

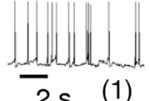

(1)

(2)

(3)
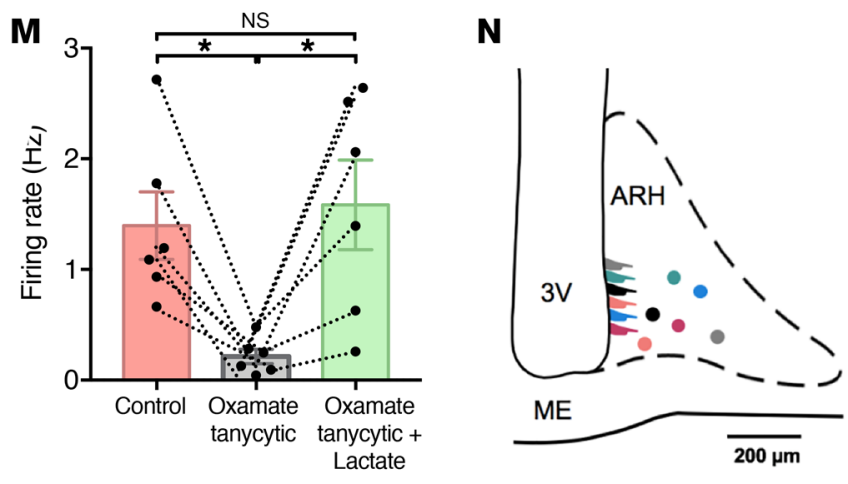

J

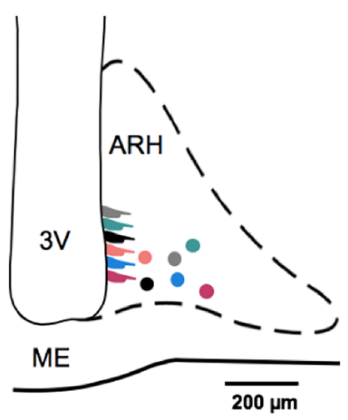

(2)

(3) 
Figure 2. Lactate supply by tanycytes sustains the activity of POMC neurons. (A and $\mathbf{B}$ ) Representative images showing vimentin (green, $\mathbf{A}$ and $\mathbf{B}$ ), MCT1 (red, A), and MCT4 (red, B) immunoreactivities in primary cultures of tanycytes. DAPI counterstaining is shown in blue (B). Scale bars: $10 \mu \mathrm{m}$. (C) Lactate secretion in cultured tanycytes exposed to different conditions (no treatment [CtrI], 2-DC, CBX, or 4-CIN). (D) Intracellular lactate in cultured tanycytes exposed to different conditions. (E) IR-DIC (left panel) and fluorescence (right panel) images of the ARH at the level of the ME showing a recording from a paired tanycyte and POMC neuron (dyad), filled with lucifer yellow (LY, green) and Alexa Fluor 594 (red), respectively. Scale bars: $50 \mu \mathrm{m}$. (F) Responses of a POMC neuron and an ARH tanycyte to a current modulation from $-50 \mathrm{pA}$ to $0 \mathrm{pA}$ with a step of $10 \mathrm{pA}$ and a duration of 1 second, and to a voltage modulation from $-100 \mathrm{mV}$ to $100 \mathrm{mV}$ with a step of $10 \mathrm{mV}$ and a duration of $300 \mathrm{~ms}$, respectively. (G-J) Schematic model (C) and a representative recording (H) of an ARH tanycyte-POMC neuron dyad (J) in whole-cell patch-clamp mode showing that dialyzing a tanycyte with $5 \mathrm{mM}$ lactate prevented the inhibitory effect of exogenous glucose deprivation ( $0 \mathrm{mM}$ glucose [0 Glc]) on neuronal firing ( $\mathbf{H}$ and $\mathbf{I})$. In the same neuron, the inhibition of MCTs by the bath application of 4-CIN cancelled this effect ( $\mathbf{H}$ and $\mathbf{I})$. Bottom traces show expansions of the recording at the time points 1, 2, 3, and 4 indicated in the trace above. (K-N) Schematic model (K) and representative recording (L) of an ARH tanycyte-POMC neuron dyad $(\mathbf{N})$ in whole-cell patch-clamp mode showing that dialyzing the tanycyte with $10 \mathrm{mM}$ oxamate inhibited POMC neuronal firing ( $\mathbf{L}$ and M). Bottom traces show expansions of the recording at the time points 1 , 2 , and 3 indicated in the trace above. In the same neuron, this inhibition was compensated for by the bath application of $5 \mathrm{mM}$ lactate ( $\mathbf{L}$ and $\mathbf{M}$ ). ${ }^{*} P<0.05$, ${ }^{* *} P<0.01$, and ${ }^{* * *} P<0.001$, by ordinary 1-way ANOVA followed by Tukey's post hoc test (C and $\mathbf{D}$ ) and repeated-measures 1-way ANOVA followed by Tukey's post hoc test (I and M) (see Supplemental Table 1 for statistical details).

sion of pyruvate to lactate, it induced a drastic reduction in action potential firing in the paired POMC neuron $(P=0.0143,1$-way, repeated-measures ANOVA followed by Tukey's post hoc test, $n=$ 6 cells, $n=5$ mice), an effect that was rescued by bath application of lactate $(P=0.0474, n=6$ cells, $n=5$ mice; Figure 2 , L-N). Altogether, these results suggest that tanycytes metabolize glucose into lactate for delivery to the POMC neurons with which they are functionally connected and that this tanycytic lactate production is both necessary and sufficient for the tonic firing of POMC neurons. In addition, the rescue of POMC neuronal activity by tanycyte-delivered lactate was completely suppressed by $500 \mu \mathrm{M} 4$-CIN ( $P=0.0288, n=6$ cells, $n=5$ mice; Figure $2, \mathrm{H}$ and $\mathrm{I}$ ), indicating that lactate transport through MCTs is required for this effect.

Mct1 and Mct4 expression by tanycytes is required to maintain POMC neuronal activity and energy homeostasis. To determine the expression profile of Mct in both tanycytes and POMC neurons, we performed quantitative PCR (qPCR) analyses in cells isolated by FACS. We collected POMC neurons from Pomc::Cre tdTomato ${ }^{\text {loxP }}$. STOP-loxP mice (Figure 3A and Supplemental Figure 2, $\mathrm{A}-\mathrm{C}$ ) and tanycytes from WT mice infused with the AAV1/2 vector expressing GFP under the tanycyte-specific iodothyronine deiodinase 2 (Dio2) promoter (27) into the lateral ventricle (Figure 3A and Supplemental Figure 2, D and F). We found that tanycytes were enriched in $M c t 1$ ( $P=0.0009$, unpaired Student's $t$ test, $n=5$ and $n=4$ mice $)$ and Mct4 transcripts ( $P=0.0272$, unpaired Student's $t$ test, $n=5$ and 4 mice), whereas POMC neurons were rich in Mct 2 mRNA ( $P=0.0028$, unpaired Student's $t$ test, $n=5$ and $n=4$ mice) (Figure 3B). To determine the relative physiological roles of these MCTs in the function of POMC neurons, we first blunted the expression of Mct2 in POMC neurons by injecting into the ARH of Pomc::Cre mice a Cre-dependent FLEXon viral vector expressing an shRNA to silence $M c t 2$. The expression of Mct2 shRNA specifically in POMC neurons (Supplemental Figure 3A) led to a clear increase in cumulative food intake (Supplemental Figure 3B) and a transient trend toward increased weight gain (Supplemental Figure 3C), in keeping with their anorexigenic function and the effect of blocking lactate uptake by these neurons on their activity seen in Figure 2, G-I. Next, we focused on tanycytic MCTs by coinjecting a Cre-dependent FLEXon viral vector expressing shRNAs to silence $M c t 1$ and Mct4 and a Dio2:Cre vector into the lateral ventricle of WT or $t d$ Tomato $^{\text {POMC }}$ mice to target tanycytes. FACS isolation of viral GFP-expressing cells showed that the Dio2:Cre-mediated expression of shRNAs targeting Mct1 and Mct4 decreased the expression of these gene transcripts by 2-fold selectively in tanycytes (ordinary 1-way ANOVA followed by Tukey's post hoc test; $M c t 1, P=0.0231 ; M c t 4, P=0.0320 ; n=4$ mice), but not in non-tanycytic cells (Mct1: $P=0.8494$, Mct4: $P=0.8944, n=4$ mice; Figure 3, C and D). While Mct1 and Mct4 knockdown (KD) in tanycytes $\left(M c t 1 / 4^{\text {TanycyteKD }}\right)$ had no effect on the expression of tanycytic markers (unpaired Student's $t$ test: vimentin, $P=0.2256$; Dio2, $P=0.4034 ; n=4$ mice; Supplemental Figure 4, A and B) or on other genes related to glucose metabolism or transport in tanycytes (unpaired Student's $t$ test: $M c t 2, P=0.8204$; Ldha, $P=$ $0.5561 ;$ Ldhb,$P=0.5457 ;$ Gck, $P=0.1381 ;$ Glut $1, P=0.2479 ;$ Glut4, $P=0.9174 ; C x 30, P=0.7825 ; C x 43, P=0.4315 ; n=6$ mice; Supplemental Figure $4 \mathrm{C})$, it markedly dampened spontaneous neuronal firing in POMC neurons $(P=0.0026$, Mann-Whitney $U$ test $; n=26$ cells, $n=5$ mice; Figure $3, \mathrm{E}-\mathrm{G})$.

Interestingly, Mct1/4 TanycteKD mice gained significantly more weight than did mice injected with the virus expressing scrambled shRNAs ( $P=0.0334$, unpaired Student's $t$ test, $n=7$ mice per group) $\left(\mathrm{Mct} 1 / 4^{\text {Tanscramble }}\right.$ F Figure $\left.3 \mathrm{H}\right)$. However, surprisingly, this weight gain was not associated with increased food intake (2-way ANOVA followed by an uncorrected Fisher's least significant difference [LSD] test; dark phase, $P=0.2454$; light phase, $P=0.8866$; mean, $P=$ 0.360 [bar graph] and $P=0.2310$ [line graph]; $n=6 \mathrm{Mct} 1 / 4^{\text {Tanscramble }}$ mice and $n=8 \mathrm{Mct1} / 4^{\text {TanycoteKD }}$ mice) (Figure 3I, see also Supplemental Figure 4D). Furthermore, although these tanycytic Mct1/4-KD mice showed only mild perturbations in energy expenditure $(P=$ 0.04480, 2-way ANOVA followed by an uncorrected Fisher's LSD test, $n=7 \mathrm{Mct} 1 / 4^{\text {Tanscramble }}$ mice and $n=8 \mathrm{Mct} 1 / 4^{\text {TanycyterD }}$ mice; Supplemental Figure 4E), they displayed a notable loss of both the linear correlation between food intake and body weight (BW) (Pearson's correlation; $M c t 1 / 4^{\text {Tanscramble }}, r^{2}=0.8087, P=0.0378 ; M c t 1 / 4^{\text {TanycyteKD }}, r^{2}$ $=0.08139, P=0.5351 ; n=7$ mice per group; Figure $3 \mathrm{~J}$ ) and between energy expenditure and BW in Mct1/4 $4^{\text {TanycytekD }}$ mice (Pearson's correlation; Mct1/4 $4^{\text {Tanscramble }}, r^{2}=0.7796, P=0.0084 ; M c t 1 / 4^{\text {TarycyterD }}$, $r^{2}=0.2481, P=0.2553 ; n=7$ mice per group; Figure $\left.3 \mathrm{~K}\right)$. Together, these results demonstrate that the maintenance of energy balance is impaired by the reduced tanycytic expression of Mct1 and Mct4.

Given the disconnect between BW and both food intake and energy expenditure observed above, we further attempted to analyze their respective components to understand the contribution of tanycytic MCTs. An analysis of ambulatory activity showed that, while horizontal displacement in the cage appeared to be unchanged between $M c t 1 / 4^{\text {TanvorterD }}$ and $M c t 1 / 4^{\text {Tanscramble }}$ mice $(P=$ 


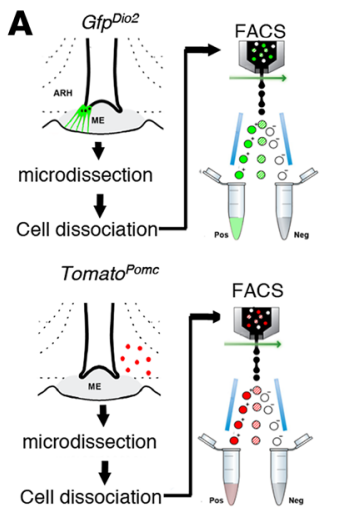

$\mathbf{E}$

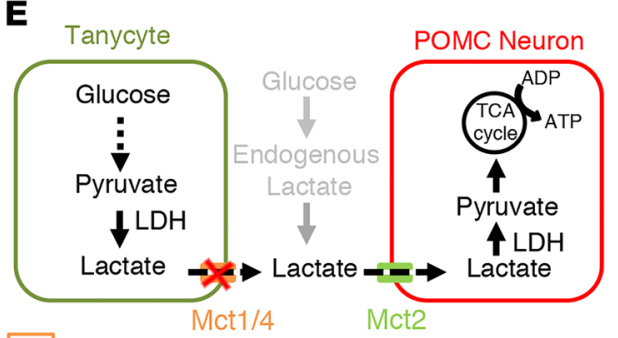

X Mct1/4 knockdown in tanycytes
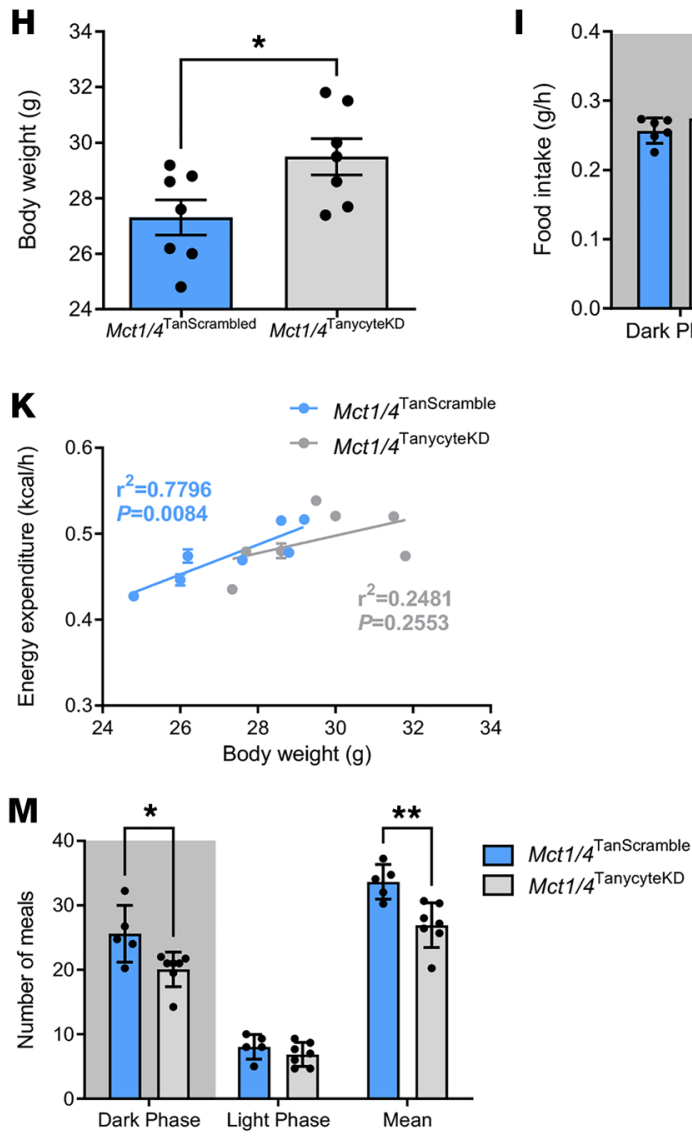

C

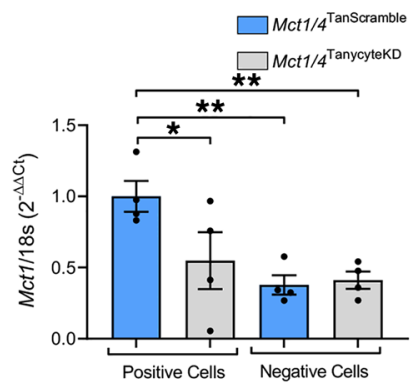

F Mct1/4Tanscramble: $t d$ Tomato ${ }^{\text {POMC }}$ $2.5 \mathrm{mM}$ Glucose
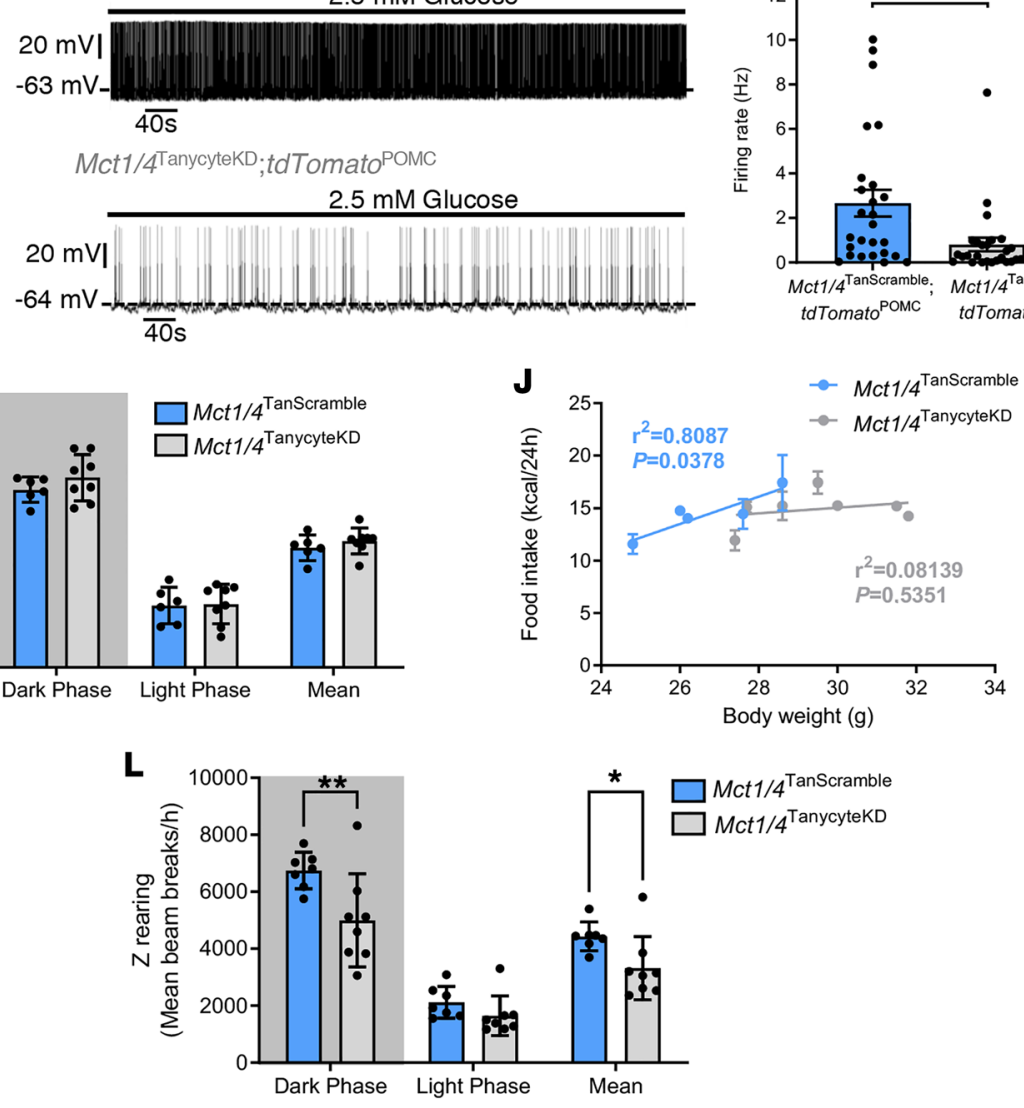

$\mathbf{N}$

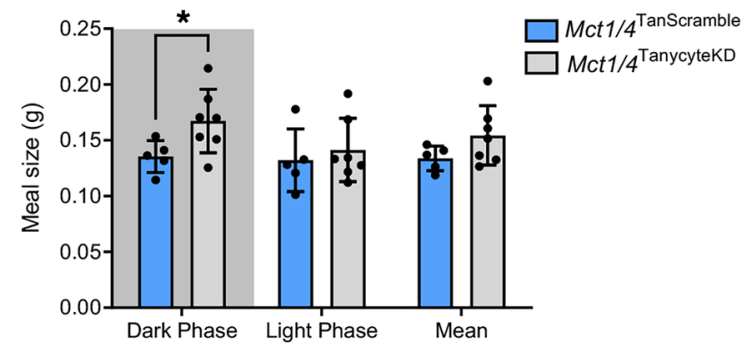


Figure 3. Inhibition of lactate transport in tanycytes alters energy

balance. (A) Schematic model for FACS used to isolate putative GFP- and Tomato-positive tanycytes and POMC neurons, respectively. (B) Relative expression of Mct1, Mct2, and Mct4 in tanycytes and POMC neurons (gene expression levels were normalized to the levels in POMC neurons, arbitrarily set at 1 ). See also Supplemental Figure 2 for characterization of the sorted cells. (C and D) Mct7 (C) and Mct4 (D) mRNA expression levels in GFP-positive and -negative cells in Mct1/4 $4^{\text {Tanscramble }}$ and Mct1/4 $4^{\text {TanycytekD }}$ mice. (E) Schematic model representing the inhibition of Mct1 and Mct4 in tanycytes. (F) Whole-cell current-clamp recordings performed in ACSF containing $2.5 \mathrm{mM}$ glucose showing the spontaneous firing rate of a POMC neuron from an Mct1/4 $4^{\text {TanycytekD }}$ mouse and another from an Mct1/4 Tanscramble mouse. (C) Comparison of firing rates of POMC neurons between Mct1/4 $4^{\text {Tanscramble }}$ tdTomato ${ }^{\text {POMC }}$ and Mct1/4 ${ }^{\text {TanycyteKD }}$ tdTomato ${ }^{\text {POMC }}$ mice. (H) BWs of Mct1/4 $4^{\text {TanScramble }}$ and Mct1/4 $4^{\text {TanycytekD }}$ mice. (I) Food intake by Mct1/4 Tanscramble and Mct1/4 $4^{\text {TanycyteKD }}$ mice during the dark and light phases. (J) Linear regression between food intake and BW for Mct1/4 Tanscramble and Mct1/4 TanycyteKD mice. (K) Linear regression between energy expenditure and BW for Mct1/4 $4^{\text {Scrambled }}$ and $M c t 1 / 4^{\text {TanycytekD }}$ mice. (L-N) Z-rearing (L), number of meals $(\mathbf{M})$, and meal size $(\mathbf{N})$ during the dark and light phases for Mct1/4 Tanscramble and Mct1/4 TanycyteKD mice. ${ }^{*} P<0.05$, ${ }^{* *} P<0.01$, and ${ }^{* *} P$ $<0.001$, by 2-tailed unpaired $t$ test (B and $\mathbf{H}$ ), Mann-Whitney $U$ test (G), ordinary 1-way ANOVA followed by an uncorrected Fisher's LSD test (C and D), 2-way ANOVA followed by an uncorrected Fisher's LSD test (I and $\mathbf{L}-\mathbf{N}$ ), and Pearson's correlation (J and $\mathbf{K}$ ).

0.5215, 2-way ANOVA followed by an uncorrected Fisher's LSD test; $n=7 \mathrm{Mct} 1 / 4^{\text {TanScramble }}$ mice and $n=8 \mathrm{Mct} 1 / 4^{\text {TanycyteKD }}$ mice; Supplemental Figure 4F), Z-rearing was significantly diminished in $M c t 1 / 4^{\text {TanycyteKD }}$ mice during the dark phase (2-way ANOVA followed by an uncorrected Fisher's LSD test: dark phase, $P=0.0012$; light phase, $P=0.3476$; mean, $P=0.0324$ (bar graph) and $P=0.0305$ (line graph); $n=7 \mathrm{Mct} 1 / 4^{\text {Tanscramble }}$ mice and $n=8 \mathrm{Mct} 1 / 4^{\text {TanycyteKD }}$ mice; Figure 3L, see also Supplemental Figure $4 G$ ). This suggests that feeding patterns may differ between these 2 experimental groups despite similar cumulative food intake (Figure 3I and Supplemental Figure 4D). Indeed, an analysis of feeding patterns showed that $M c t 1 / 4^{\text {TanycyteKD }}$ mice ate less frequently than did $M c t 1 / 4^{\text {Tanscramble }}$ mice (2-way ANOVA followed by an uncorrected Fisher's LSD test: dark phase, $P=0.0459$; light phase, $P=0.3042$; mean, $P=0.0037 ; n=5$ $M c t 1 / 4^{\text {Tanscramble }}$ mice and $n=7 \mathrm{Mct} 1 / 4^{\text {TanycyteKD }}$ mice; Figure $\left.3 \mathrm{M}\right)$, but that the size of their meals was significantly increased (2-way ANOVA followed by an uncorrected Fisher's LSD test: dark phase, $P=$ 0.0337; light phase, $P=0.5919$; mean, $P=0.1018 ; 5 \mathrm{Mct} 1 / 4^{\text {Tanscramble }}$ mice and $n=7 \mathrm{Mct} 1 / 4^{\text {TanycyteKD }}$ mice; Figure $\left.3 \mathrm{~N}\right)$.

Altogether, these data suggest that tanycytic lactate shuttles control the activity of POMC neurons and hence contribute to the adaptation of the feeding pattern of the individual to energy needs, i.e., glucose availability.

POMC neuronal activity depends on gap junction-mediated tanycytic metabolic networks. Intriguingly, in the paired tanycyte-neuron recording setup illustrated in Figure 2E, Lucifer yellow filling the patch pipette diffused from the patched tanycyte into neighboring tanycytes along the wall of the $3 \mathrm{~V}$. This phenomenon, which we also observed when using biocytin-filled patch pipettes (Supplemental Figure 5, A and B), suggests the existence of a functional tanycytic network in the ARH, similar to the gap junction-mediated astrocytic networks previously described in the brain (28), including in the lateral hypothalamic area (24). The intercellular diffusion of biocytin was blunted by the bath appli- cation of the gap junction blocker carbenoxolone (CBX, $50 \mu \mathrm{M})$ (Supplemental Figure 5, C and D, and ref. 28), suggesting that tanycytes are also connected by gap junctions. Under control conditions, biocytin diffused through the cell bodies of ARH tanycytes over 100-200 $\mu \mathrm{m}$ along the wall of the $3 \mathrm{~V}$ and into tanycytic processes extending into the tissue, but not into cells expressing the neuronal marker NeuN (Supplemental Figure 5B). In the presence of CBX (50 $\mu \mathrm{M}, 30 \mathrm{~min})$, recorded POMC neurons showed a marked decrease in their firing activity $(P=0.0312$, Wilcoxon matched-pairs test, $n=6$ cells, $n=4$ mice; Figure 4 , A-C), an effect that could be prevented by bath application of exogenous lactate ( $P=0.625$, Wilcoxon matched-pair test, $n=5$ cells, $n=4$ mice; Figure $4, \mathrm{D}$ and $\mathrm{E})$. Interestingly, the treatment of cultured tanycytes with CBX phenocopied the effects of 4-CIN, i.e., it also prevented the release of lactate into the conditioned medium $(P=0.0355$, 1-way repeated-measures ANOVA followed by Tukey's post hoc test, $n=6$; Figure 2C) and led to its accumulation in the cytoplasm of tanycytes ( $P=0.0009,1$-way, repeated-measures ANOVA followed by Tukey's post hoc test, $n=6$; Figure 2D). Together, these results demonstrate functional gap-junctional coupling between tanycytes surrounding POMC neurons in the ARH and raise the possibility that such glial metabolic networks play a role in the regulation of POMC neuronal activity that is greater than the sum of the roles of individual tanycytes.

In agreement with previous studies $(29,30)$, we found that tanycytes expressed the gap junction protein connexin 43 (Cx43), with a marked enrichment in ARH tanycytes when compared with median eminence (ME) tanycytes, which are responsible for the transport of glucose and other peripheral metabolic signals into the CSF (refs. 22, 25, 31 and Supplemental Figure 5, E-G). To determine the functional role of these Cx43-mediated tanycytic networks in the control of POMC neuron activity, we next selectively knocked out the Gja1 gene encoding $\mathrm{Cx} 43$ in tanycytes by infusing the recombinant TAT-Cre protein into the $3 \mathrm{~V}$ of $C x 43^{\text {loxp/loxp }}$ mice (Figure $4 \mathrm{~F}$ ), a procedure that we have extensively characterized before $(4,32)$. FACS isolation of TAT-Creinjected $\mathrm{Cx}_{4} 3^{+/+}$tdTomato ${ }^{\text {loxP-STOP-loxP }}\left(\mathrm{Cx} 43^{+/+}\right.$tdTomato $\left.^{\text {Tanycyte }}\right)$ and $C x 43^{\text {loxP/loxP }}$ tdTomato ${ }^{\text {loxp-STOP-loxP }}$ (Cx43 $3^{\text {TanycyteKO }}$ tdTomato ${ }^{\text {Tanycyte }}$ ) mice (Supplemental Figure 6), demonstrated that $C x 43$ expression was significantly diminished in Tomato-positive tanycytes $(P$ $=0.0449$, unpaired Student's $t$ test, $n=6$ mice; Figure 4G), but not in Tomato-negative cells ( $P=0.8611$, unpaired Student's $t$ test, $n=$ 6 mice; Figure $4 G$ ), which comprise astrocytes that are also known to abundantly express $\mathrm{Cx} 43$ (24), in floxed $\mathrm{Cx} 43$ mice. In line with our recent data showing that our TAT-Cre infusion procedure causes genetic recombination in approximately $60 \%$ of ME/ARH tanycytes (33), Cx43 protein expression was fully extinguished in the subset of tanycytes expressing high levels of Tomato (Supplemental Figure 5I). This submaximal invalidation of $\mathrm{Cx} 43$ protein expression in tanycytes was sufficient to abolish the gap junction-mediated tanycytic network, as shown by the absence of intercellular diffusion following biocytin injection into a single ARH tanycyte via a patch pipette $(P=0.0006$, Mann-Whitney $U$ test, $n=7$ cells from $n=6 C x 43^{\text {loxP/loxP }}$ mice and $n=7$ cells from $n=6 C x 43^{\text {TanycyteKO }}$ mice; Supplemental Figure 5, J and K). Consequently, the selective deletion of $\mathrm{Cx} 43$ in tanycytes markedly diminished the ability of energy metabolites to be trafficked between ARH tanycytes, as shown 
A
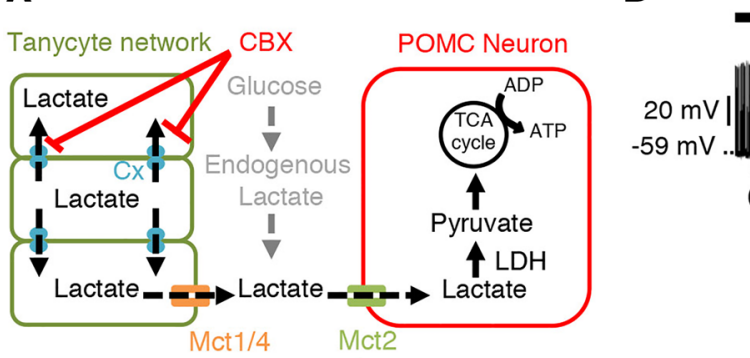

D

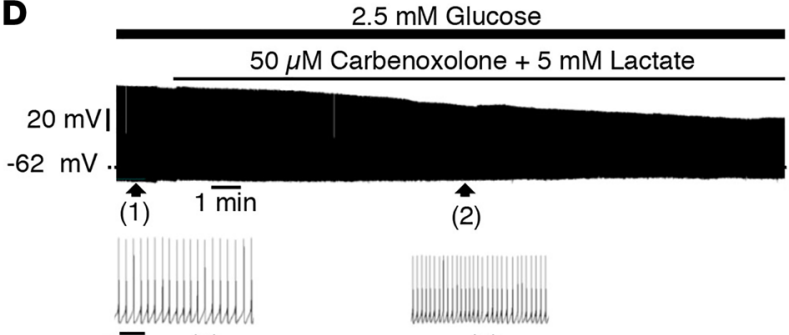

$1 \overline{\mathrm{sec}}(1)$

G
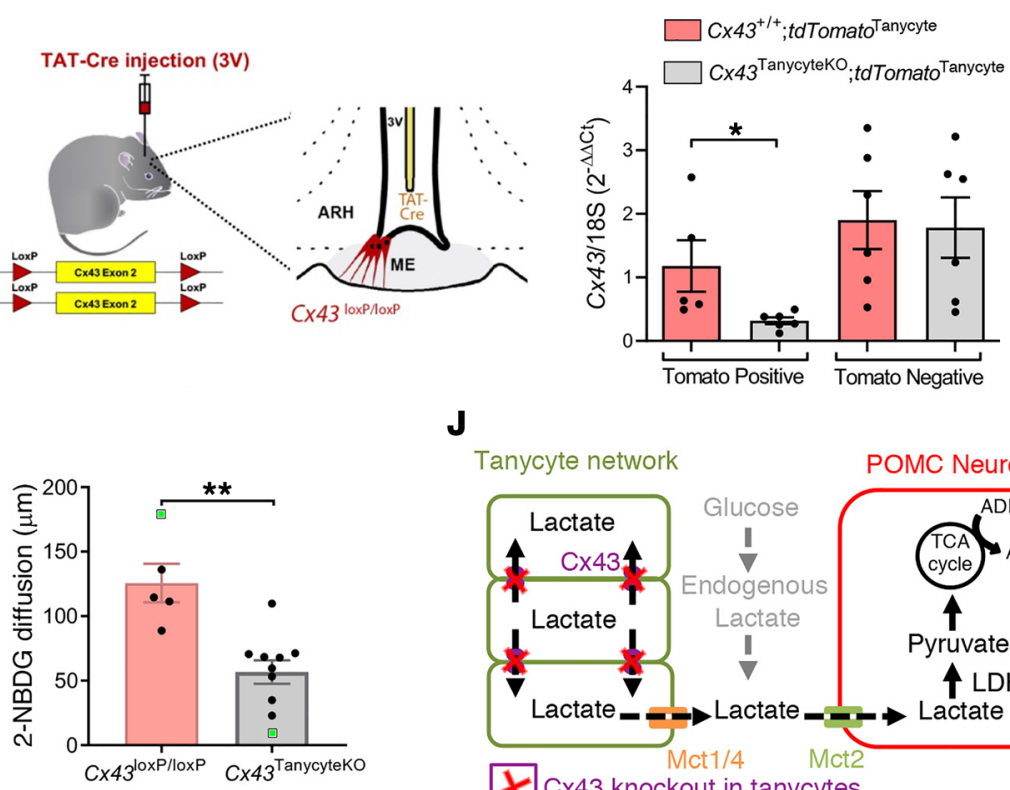

$\mathbf{L}$

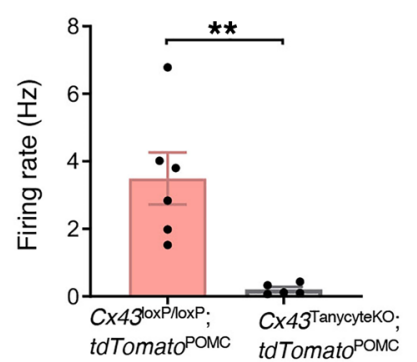

M

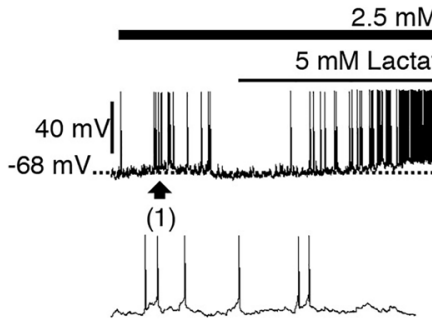

(1)
J

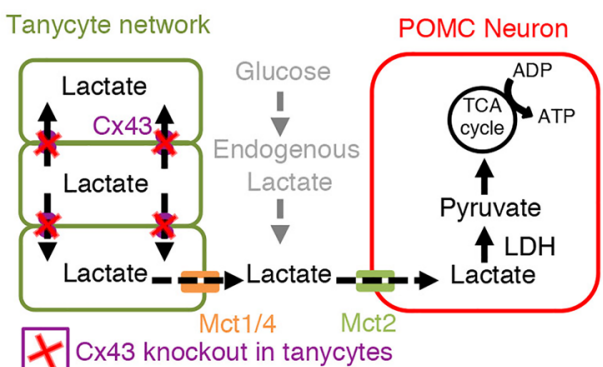

XCx43 knockout in tanycytes
(2)

H

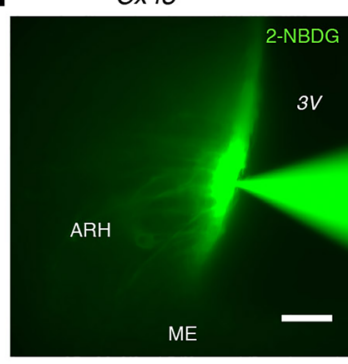

(2)

(2)
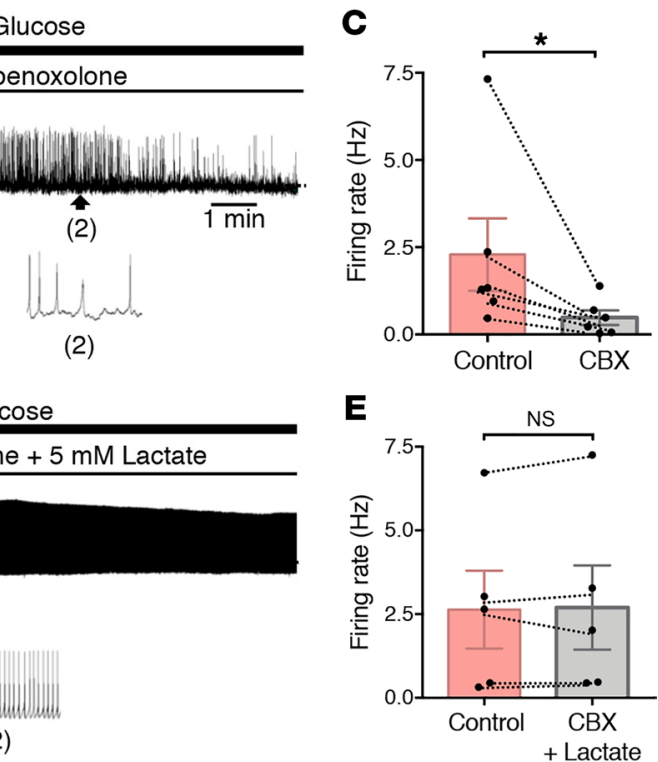

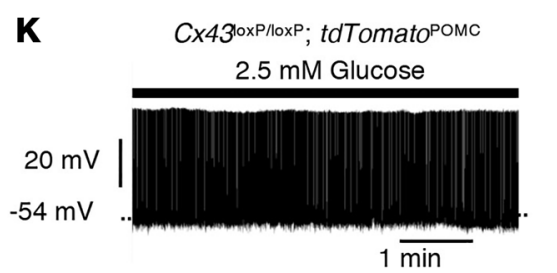

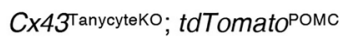
$2.5 \mathrm{mM}$ Glucose

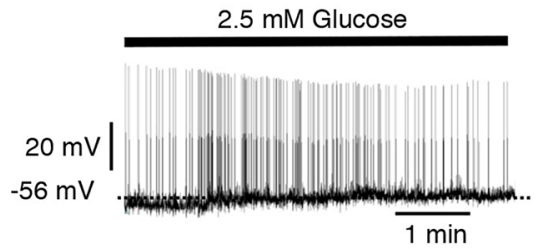

$\mathbf{N}$

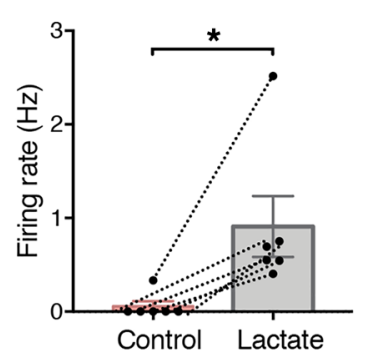


Figure 4. Lactate trafficking through the tanycytic network is necessary to sustain POMC neuronal activity. (A) Schematic model representing the involvement of the tanycytic network in shuttling lactate to POMC neurons. CX, connexins. (B-E) Representative whole-cell current-clamp recording of a POMC neuron (B and $\mathbf{D}$ ) in ACSF containing a physiological concentration of glucose $(2.5 \mathrm{mM})$, showing that the bath application of CBX decreased the spontaneous firing rate of the neuron (B and $\mathbf{C}$ ) and that bath application of lactate reversed this effect ( $\mathbf{D}$ and $\mathbf{E})$. (F) $C x 43^{\text {TanyyyteKo }}$ model. Tanycyte-specific deletion of $C x 43$ was carried out by infusing the TAT-Cre fusion protein into the $3 \mathrm{~V}$ of $C \times 43^{10 \times P / 10 \times P}$ mice. (C) mRNA expression levels of $C \times 43$ in Tomato-positive and -negative cells in $\mathrm{C} \times 43^{+/+}$and $\mathrm{C} \times 43^{\text {Tanycyteko }}$ mice. (H and I) Images (H) showing the maximal and minimal diffusion through ARH tanycytes of the fluorescent glucose analog 2-NBDG (green) injected into a single tanycyte in a control $C \times 43^{10 \times P / 10 \times P}$ mouse and a $C \times 43^{\text {TanycteKO }}$ mouse, respectively, via a patch pipette for 20 minutes $(\mathbf{H})$, and quantification of the diffusion (I); the 2 animals highlighted in green in the quantitative data in I correspond to the extremes shown in $\mathbf{H}$. Scale bars: $50 \mu \mathrm{m}$. (J) Schematic model representing the involvement of a tanycytic network mediated by $C \times 43$ gap junctions in lactate shuttling to POMC neurons. This model was tested by recording the electrical activity of POMC neurons in $C \times 43^{\text {TanyyyteKO }}$ mice. ( $\mathbf{K}$ and $\mathbf{L}$ ) Representative whole-cell current-clamp recording performed in ACSF containing $2.5 \mathrm{mM}$ glucose showing the spontaneous firing rate of a POMC neuron from a $C \times 43^{\text {loxP/IOXP }}$ tdTomato ${ }^{\text {POMC }}$ (top trace) and a Cx43 $3^{\text {TanycyteKo }}$ tdTomato ${ }^{\text {POMC }}$ (top trace) mouse, as quantified in $\mathbf{L}$. ( $\mathbf{M}$ and $\mathbf{N}$ ) Representative whole-cell current-clamp recording of a POMC neuron from a $C \times 43^{\text {TanycyteKo }} t d$ Tomato ${ }^{\text {POMC }}$ mouse performed in ACSF containing $2.5 \mathrm{mM}$ glucose, showing that the bath application of $5 \mathrm{mM}$ lactate increased its firing rate, as quantified in $\mathbf{N}$. ${ }^{*} P<$ 0.05 and ${ }^{* *} P<0.01$, by Wilcoxon matched-pairs test (C, E, and $\mathbf{N}$ ), 2-tailed, unpaired Student's $t$ test (G and I), and Mann-Whitney $U$ test (L).

by the injection of a single tanycyte with the fluorescent glucose derivate 2-(N-[7-nitrobenz-2-oxa-1,3-diazol-4-yl]amino)-2-deoxyglucose (2-NBDG, $2 \mathrm{mg} / \mathrm{mL}$, ref. 28 , and Figure $4, \mathrm{H}$ and I) $(P=$ 0.0011 , unpaired Student's $t$ test, $n=5$ cells from $n=2 C x 43^{\text {loxP } / \text { loxP }}$ mice and $n=10$ cells from $n=4 C x 43^{\text {TanycrteKO }}$ mice). This deletion of $\mathrm{Cx} 43$ specifically in tanycytes also decreased spontaneous POMC neuronal firing $(P=0.0043$, Mann-Whitney $U$ test, $n=6$ cells from $n=C x 43^{\text {loxP/loxP }}$ mice and $n=5$ cells from $n=4 C x 43^{\text {TanycyteKO }}$ mice; Figure 4, J-L), which was restored by the bath application of 5 $\mathrm{mM}$ lactate in the presence of $2.5 \mathrm{mM}$ glucose $(P=0.0312$, Wilcoxon matched-pairs test, $n=6$ cells, $n=5$ mice; Figure $4, \mathrm{M}$ and $\mathrm{N}$ ). However, in $C x 43^{\text {loxP/loxp }}$ littermates, the bath application of lactate had no effect on POMC neuronal activity, which was normal $(P=0.6042$, paired Student's $t$ test, $n=6$ cells, $n=6$ mice; Supplemental Figure 5 , $\mathrm{L}$ and $\mathrm{M}$ ). These results suggest that the decreased activity of POMC neurons in $C x 43^{\text {TanycyteKO }}$ mice resulted from a deficiency in the trafficking of glucose-derived lactate through the tanycytic network.

Tanycytic Cx43 KO impairs changes in ARH lactate levels in response to blood glucose variation. CSF glucose levels, which are maintained at approximately $60 \%$ of serum glucose levels (11, 12 ), have long been proposed to complete a feedback loop in the homeostatic control of food intake and BW (34). Regardless of its source, this role of glucose in energy homeostasis appears to require the production of lactate by glial cells in the hypothalamus (17), and central lactate metabolism has been shown to regulate food intake $(35,36)$. We thus next assessed the putative involvement of gap junction-mediated tanycytic metabolic networks, which metabolize glucose into lactate (Figure 2, C, D, and K-M), in controlling the bioavailability of lactate in hypothalamic tissue in a physiological context. Microdialysis experiments in the ARH of $C x 43^{\text {loxP/loxP }}$ mice and littermates in which $C x 43$ was knocked out $\left(C x 43^{\text {TanycyteKO }}\right)$ using either the TAT-Cre recombinant protein or the Dio2:Cre viral approach (see Figure 5C, right panel for a comparison of the 2 methods), before and after i.p. injection of saline and glucose ( $2 \mathrm{~g} / \mathrm{kg} \mathrm{BW}$; Figure $5, \mathrm{~A}-\mathrm{C})$ showed that, while saline injection did not alter lactate levels (2-way repeated-measures ANOVA followed by an uncorrected Fisher's LSD test, $n=6 C x 43^{\text {loxp } / l o x P}$ and $n=10 \mathrm{Cx} 43^{\text {TanycteKO }}$ mice; vehicle, $40 \mathrm{~min}$ vs. basal, $P=0.6663$ for $C x 43^{\text {loxP/loxP }}$ mice and $P=0.3059$ for $C x 43^{\text {TanycyteKO }}$ mice $\backslash$ ), glucose injection quickly promoted an increase in the hypothalamic levels of lactate in $C x 43^{\text {loxP/loxP }}$ mice $(P=0.0008$ : vehicle, 20 min vs. glucose, $20 \mathrm{~min} ; P<0.0001$ : vehicle, $40 \mathrm{~min}$ vs. glucose, $40 \mathrm{~min})$. In contrast, we found that peripheral glucose injection had no effect on hypothalamic levels of lactate in $C x 43^{\text {TапусуteKO }}$ mice $(P=0.1153$ : vehicle, $20 \mathrm{~min}$ vs. glucose, $20 \mathrm{~min} ; P=0.0049$ : vehicle, $40 \mathrm{~min}$ vs. glucose, $40 \mathrm{~min}$ ), which remained lower than in $C x 43^{\text {loxp } / \text { loxp }}$ animals after glucose injection $(P=0.0012$, at $40 \mathrm{~min})$. Our results suggest that tanycytic metabolic networks along the wall of the $3 \mathrm{~V}$ that are in contact with both the blood circulation and the CSF are actively involved in integrating the changes in circulating glucose levels and translating these changes into a local lactate signal that can be perceived by ARH neurons and used to drive their activity. Furthermore, downregulation of the expression of the glucose transporter Glut2 ( $P=0.0071$, unpaired Student's $t$ test, $n=6$ mice $)$ and the lactate dehydrogenase Ldhb $(P=0.0119$, unpaired Student's $t$ test, $n=6$ mice) in tanycytes with Cx43 KO (Figure 5D) may further accentuate the shortage of tanycytic lactate supply to ARH neurons in mutant mice.

KO of Cx43 in ARH tanycytes causes weight gain and modulates substrate consumption. Supporting the role of the glucose-sensitive tanycytic network in modulating the activity of POMC neurons controlling food intake in response to the peripheral energy state, $C x 43^{\text {ТапусуteкO }}$ mice had increased food intake $(P=0.0428$, unpaired Student's $t$ test, $n=7 C x 43^{\text {loxP/loxP }}$ and $n=9 C x 43^{\text {TanycyteKO }}$ mice; Figure 5E), BW ( $P=0.0121$, unpaired Student's $t$ test, $n=9$ mice per group; Figure $5 \mathrm{~F})$, and fat mass content $(P=0.0287$, unpaired Student's $t$ test, $n=8 C x 43^{\text {loxP/loxP }}$ and $n=9 C \times 43^{\text {TanycyteKO }}$ mice; Figure $5 G)$, but no change in lean mass $(P=0.1139$, Mann-Whitney $U$ test, $n=8 C x 43^{\text {loxP/loxP }}$ and $n=9 C x 43^{\text {TanycyteKO }}$ mice; Figure $5 \mathrm{H}$ ), when compared with vehicle-injected $C x 43^{\text {loxp/loxp }}$ littermates 10 days after TAT-Cre injection into the $3 \mathrm{~V}$. Given that the central melanocortin system not only modulates feeding but also controls energy partitioning and expenditure (37-40), we further investigated the role of tanycytic $\mathrm{Cx} 43$ in the regulation of energy homeostasis. $C x 43^{\text {loxp/loxp }}$ mice were subjected to indirect calorimetry before and after TAT-Cre injection. After TAT-Cre injection, $C x 43^{\text {TanycyteKO }}$ mice, in addition to the increase in food intake noted above ( $P=0.0001$, 2-way ANOVA, uncorrected Fisher's LSD test, $n=7$ mice per group; Figure 5I), showed a decrease in ambulatory activity $(P<0.0001,2$-way ANOVA, uncorrected Fisher's LSD test, $n=7$ mice per group; Figure 5J) and energy expenditure $(P=$ 0.0001, 2-way ANOVA, uncorrected Fisher's LSD test, $n=7$ mice per group; Figure 5K). Importantly, selective $\mathrm{KO}$ of Cx43 in tanycytes also induced a marked increase in the respiratory exchange ratio (RER) ( $P=0.0147,2$-way ANOVA, uncorrected Fisher's LSD test, $n=7$ mice per group; Figure $5 \mathrm{~L}$ ) together with a decrease in oxygen consumption (Figure $5 \mathrm{M}$ ), during both the resting phase 
A

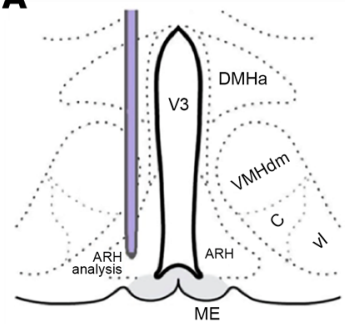

B

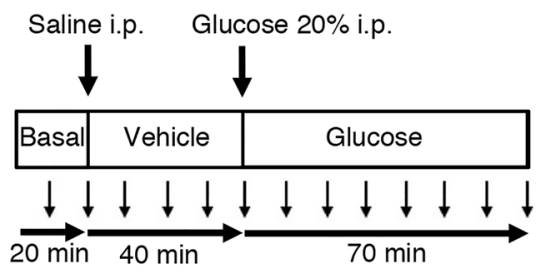

C

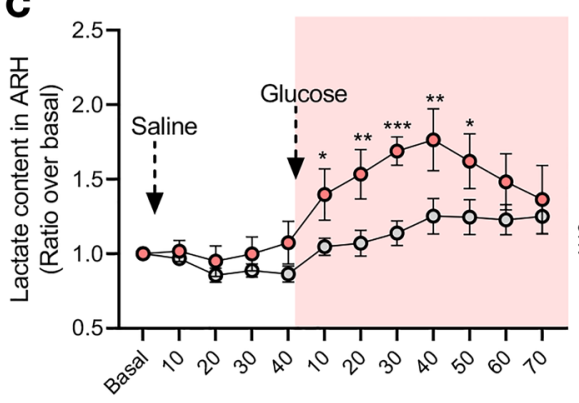

- $C \times 43^{\text {loxP/loxP }}$

- $C \times\left. 43^{\text {TanycyteKo }}\right|^{\star \star *}$

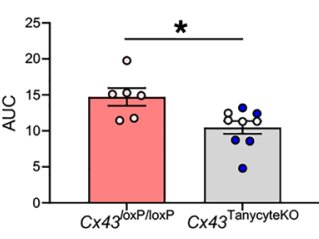

D $\square C \times 43^{+++}:$tdTomato $0^{\text {Tanvcute }}$

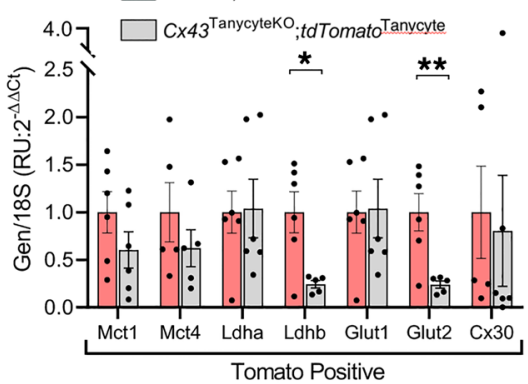

G

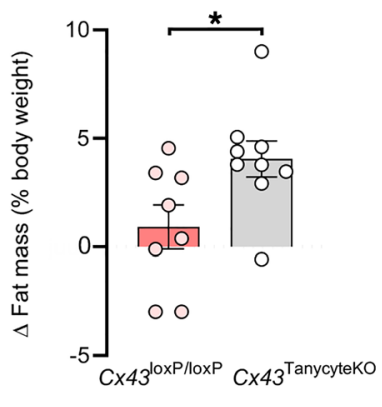

H

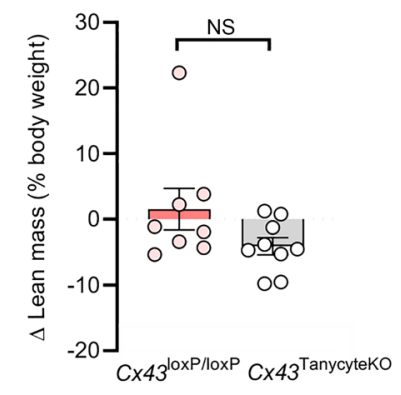

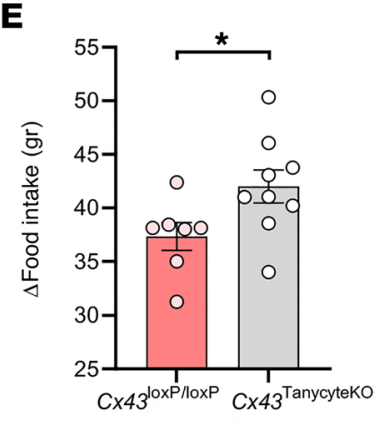
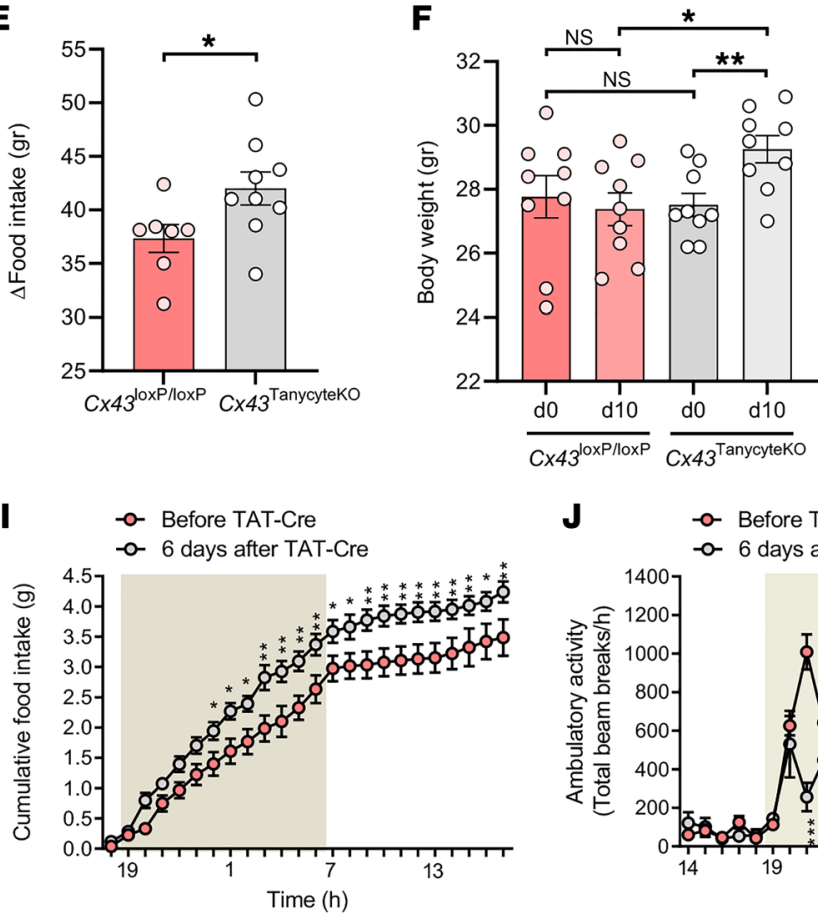

$\mathbf{L}$

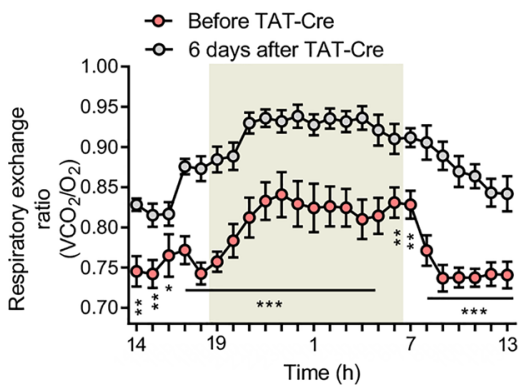

J

- - Before TAT-Cre

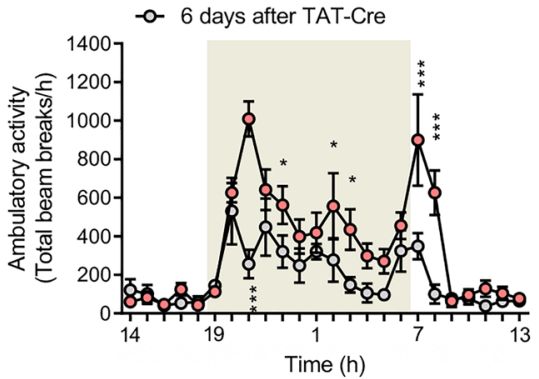

M

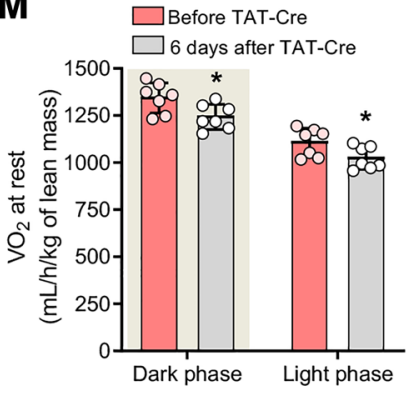

K $\quad$ - Before TAT-Cre

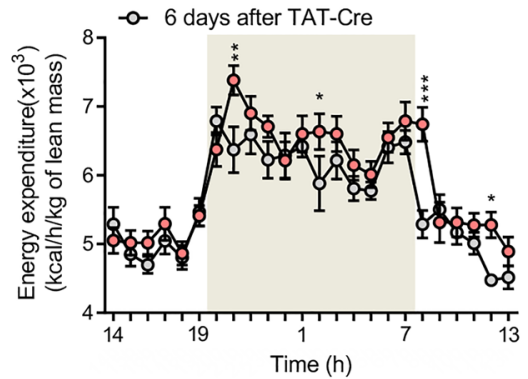

$\mathbf{N}$

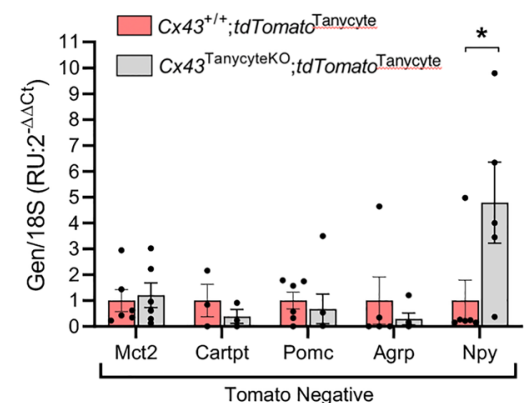


Figure 5. Lactate trafficking in the tanycytic network is necessary to sustain energy homeostasis. (A-C) Lactate levels in the mediobasal hypothalamus (C) assessed using microdialysis (A) in $C \times 43^{\text {Tanycyteko }}$ mice compared with $C \times 43^{10 \times P / / 0 x P}$ mice, before (basal) and after an i.p. injection of saline followed by glucose ( $2 \mathrm{mg} / \mathrm{kg} \mathrm{BW}$ ) (B). The bar graph in C represents the AUC during glucose treatment in $C \times 43^{\text {loxP/loxP }}$ mice or $C x 43^{\text {Tanycyteko }}$ littermates, in which gene recombination was either induced by infusing the recombinant TAT-Cre protein into the $3 \mathrm{~V}$ ( $n=4$, white dots) or injecting the AAV1/2 Dio2:Cre into the lateral ventricle ( $n=5$, black dots). (D) Comparison of mRNA expression levels of Mct1, Mct4, Ldha, Ldhb, Glut1, Glut2, and Cx30 in Tomato-positive cells between $C x 43^{+/+} t d T_{0}$ mato ${ }^{\text {Tanycyte }}$ and $C x 43^{\text {Tanycyteko }}$ tdTomato ${ }^{\text {Tanycyte }}$ mice. (E) Change in food intake between day 0 and day 10 following TAT-Cre injection into $C \times 43^{\text {Tanyyteko }}$ mice compared with $C \times 43^{\text {loxP/loxp }}$ control mice. $(\mathbf{F})$ BWs of $C \times 43^{\text {TanyyyteKo }}$ mice and $C \times 43^{\text {loxP/loxP }}$ control mice on day 0 (d0) and on day 10 (d10) following TAT-Cre injection. ( $\mathbf{G}$ and $\mathbf{H}$ ) Change in fat mass $(\mathbf{G})$ and lean mass $(\mathbf{H})$ between day 0 and day 10 following TATCre injection in $C \times 43^{\text {Tanycytek0 }}$ mice compared with $C \times 43^{\text {loxP/loxP }}$ control mice. (I-L) Cumulative food intake (I), ambulatory activity (J), energy expenditure (K), and RER (L) before and 6 days after TAT-Cre injection into the 3V. (M) $\mathrm{O}_{2}$ consumption at rest 6 days after TAT-Cre injection into the $3 \mathrm{~V}$, during the dark and light phases. (N) mRNA expression levels of Mct2, Cartpt, Pomc, Agrp, and Npy in Tomato-negative cells from $C \times 43^{+/+}$versus $C X 43^{\text {Tanycyteko }}$ mice. ${ }^{*} P<0.05$, ${ }^{* *} P<0.01$, and ${ }^{* *} P<0.001$, by 2 -way ANOVA followed by an uncorrected Fisher's LSD test (C, left graph, and I-L), 2-tailed, unpaired Student's $t$ test (C, right graph, D-F, between genotypes, and $\mathbf{G}, \mathbf{M}$, and $\mathbf{N}$ ), 2-tailed, paired Student's $t$ tests ( $\mathbf{F}$, between day 0 and day 10 within the same genotype), and Mann-Whitney $U$ test $(\mathbf{H})$.

(day; $P=0.0394$, unpaired Student's $t$ test, $n=7$ mice per group) and the active phase (night; $P=0.0261$, unpaired Student's $t$ test, $n$

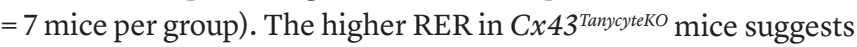
that nutrient partitioning was altered, favoring the use of carbohydrates rather than fatty acids as an energy source in these mice, contrary to the normal situation (41). In addition to a dramatic impairment of POMC neuronal activity (Figure 4, K and L), these changes in metabolism after selective $\mathrm{KO}$ of $\mathrm{Cx} 43$ in tanycytes were associated with an upregulation of transcripts for NPY in the ARH ( $P=0.0492$, unpaired Student's $t$ test, $n=6$ mice $)$, whereas transcripts for Pomc $(P=0.6335$, unpaired Student's $t$ test, $n=6$ mice), $\operatorname{Cart}(P=0.4184$, unpaired Student's $t$ test, $n=6$ mice $)$, and $\operatorname{Agrp}(P=0.4722$, unpaired Student's $t$ test, $n=6$ mice $)$ appeared to be unchanged in the mutant mice (Figure $5 \mathrm{~N}$ ). The gap junction-mediated ARH tanycytic network thus appears to be a physiologically relevant component of metabolic regulation, modulating feeding behaviors and the establishment of energy balance in response to peripheral conditions.

\section{Discussion}

The question of what energy metabolites reach POMC neurons in the ARH has a 2-fold significance: first, like every other type of neuron, POMC neurons need an energy source to drive their activity; but second, and more important, the activity of these anorexigenic neurons itself controls the energy state of the organism, making it essential for them to sense metabolic signals triggered by nutrient intake or energy consumption at the periphery.

We believe that our results shed new light on a long-established but ill-understood phenomenon $(10,42,43)$ by demonstrating that POMC neurons depend on the bioavailability of lactate for their activity and that this lactate is metabolized from glucose and distributed to neurons by a network of ARH tanycytes. Intriguing- ly, the dramatic suppression of POMC neuronal activity in mice in which lactate transfer by tanycytes was blocked indicates that, with regard to this neuronal population at least, the astrocyte-neuron lactate shuttle $(23,44)$, which up to now was thought to explain the regulation of synaptic transmission and the fueling of neuronal activity in neocortical (28) and hypothalamic brain regions (24), has been almost completely replaced by a shuttle consisting of another glial cell type, the tanycyte. This places tanycytes at the center of the intercellular communication processes used by hypothalamic circuits regulating energy homeostasis, where they play the role of bloodhounds of the metabolic brain, "sniffing" out the availability of glucose and conveying this information to neurons in such a way as to modulate their activity. Indeed, studies in human patients lacking the glucose transporter GLUT1 suggest that CSF-borne peripheral glucose is an important source of lactate in the brain (45). The peculiar position occupied by tanycytes, at the interface of both the peripheral circulation and the CSF, makes them ideal candidates for the detection and perhaps triangulation of circulating metabolic signals, potentially providing qualitative in addition to quantitative information. Importantly, the fact that the energy metabolite released by ARH tanycytes and used by POMC neurons to adjust their activity to physiological demands is lactate and not glucose provides a plausible explanation for why POMC neurons, whose function it is to respond to changes in glucose levels, do not themselves appear to be capable of sensing glucose (9). Furthermore, consistent with the notion that non-neuronal cells are functionally involved in the regulation of metabolic sensing and the central control of systemic metabolism $(33,46-48)$, recent studies have reported that insulin receptor expression in astrocytes is involved in the regulation of glucose uptake at the blood-brain barrier (46) and may participate in the glucose-induced activation of POMC neurons, possibly via changes in the glial coverage of POMC neuronal cell bodies $(46,49)$. It remains to be determined whether and how this newly uncovered role for astrocytes modulates the role of tanycytes, or vice versa, in the fine-tuning of POMC neuronal function.

Intriguingly, our findings reveal both the involvement of the monocarboxylate transporters MCT1 and MCT4 and a previously unknown role for $\mathrm{Cx} 43$ gap junction channels in the transfer of lactate to POMC neurons by ARH tanycytes. Indeed, the connexins appear to constitute the molecular basis of the newly discovered metabolic network formed by these CSF-contacting tanycytes. The ability to mobilize and transfer lactate through a large number of interconnected cells may serve to increase the sensitivity of the response to any peripheral signal beyond the sum of the responses of a number of unconnected cells, each releasing its own load of lactate. In addition to this amplification of the efficiency with which a metabolic signal reaches its targets, the existence of these gap junction-mediated tanycytic networks could improve the adaptive metabolic response by synchronizing the release of energy metabolites to neuronal populations that need to be informed about variations in physiological state.

Keeping in mind that blocking or knocking out $\mathrm{Cx} 43$ specifically in ARH tanycytes phenocopies the effect of the MCT inhibitor 4-CIN, the potential involvement of connexin hemichannels themselves in the secretion of lactate by tanycytes (50) or a signaling role for gap junctions above and beyond the transfer of 
metabolites between tanycytes cannot be ruled out. A putative signaling role for $\mathrm{Cx} 43$ in the control of tanycytic lactate secretion is suggested by our in vitro data showing that treatment of cultured tanycytes with the gap junction blocker carbenoxolone altered lactate release (Figure 2C). Our in vivo results also strengthen this possibility, as they showed that knocking out $\mathrm{Cx} 43$ in tanycytes resulted in the downregulation of 2 critical molecules specifically in these cells: the glucose transporter Glut2, which has recently been suggested to promote the rapid transfer of circulating glucose into the CSF at the ME (31), and the lactate dehydrogenase $L d h b$. Together with the recent demonstration that $\mathrm{Cx} 43$ can directly act as a transcriptional regulator (51), these results raise the intriguing possibility that $\mathrm{Cx} 43$ may form a functional micronetwork with other genes, e.g., Glut2 and Ldhb, in tanycytes, although it remains to be determined whether such a micronetwork would simply dictate the terms of glucose conversion into lactate, or would itself be subject to feedback regulation by accumulating intracellular lactate that has nowhere to go when Cx43 or the MCTs are blocked.

The physiological relevance of these results is demonstrated by the phenotype of mice lacking $\mathrm{Cx} 43$ in ARH tanycytes. Their inability to metabolize lactate from glucose and the resulting lower activity of POMC neurons cause a profound metabolic dysfunction represented by hyperphagia, increased weight gain and adiposity, decreased energy expenditure, and an increased RER, indicating a switch from lipid to carbohydrate use. It is well established that the central melanocortin system, of which the POMC neurons form an integral element, regulates all these parameters. Interestingly, our mice in which tanycytic $\mathrm{Cx} 43$ was inactivated appeared to phenocopy those lacking $\operatorname{POMC}(52,53)$ or melanocortin receptor $4(54)$. This, together with the fact that the selective impairment of $M c t 1$ and $M c t 4$ expression in tanycytes is as effective at inhibiting POMC neuronal activity as knocking out $\mathrm{Cx} 43$ in these cells, strongly suggests that the low bioavailability of tanycytic lactate shuts down the activity of POMC neurons and that this deficiency cannot be compensated by any other neuroglial pathway. Strengthening this idea are our patch-clamp experiments showing that the direct infusion of the lactate dehydrogenase inhibitor oxamate into the $\mathrm{Cx} 43$-connected tanycytic network, effectively abolishing the conversion of bath-borne glucose into lactate by tanycytes, sufficed to blunt the spontaneous firing of POMC neurons in living brain slices. In addition, the selective knockdown of $M c t 1$ and Mct4 expression in tanycytes also provided a more nuanced view of the manner in which these cells influence food intake. Indeed, the lack of tanycytic Mct1 and Mct4 not only dramatically impaired the spontaneous activity of POMC neurons, but the resulting BW gain occurred without a marked change in cumulative food intake, instead altering meal patterns and uncoupling both energy intake and energy expenditure from bodily needs, unlike the more straightforward effect observed when Mct2 alone was knocked down in POMC neurons. These results agree with previous studies showing that the activity of a subpopulation of ARH POMC neurons projecting to the brainstem controls feeding behavior and that the inhibition of melanocortinergic activity in the brainstem nucleus of the solitary tract increases meal size $(55,56)$. Another interesting question, however, is whether the tanycytic lactate shuttle or gap-junctional network is required for the activity of other hypothalamic neurons, such as the orexigenic agouti-related peptide (AgRP) and neuropeptide Y (NPY) neurons, which play a role opposite that of POMC neurons in energy homeostasis $(6,9)$, but which are also rich in MCT2 and may play a confounding role when MCT2 is silenced in POMC neurons (57). Our results indeed show that knocking out Cx43 in tanycytes upregulates NPY transcripts in the mediobasal hypothalamus. Whether this change, which may, at least in part, contribute to the increased feeding seen in mutant mice, is a direct or an indirect consequence of the impaired tanycytic lactate shuttle remains to be explored.

In conclusion, our results reveal an unexpected role for tanycytic networks connected by gap junctions, thanks to their unique position and ability to convert glucose into lactate, as both the suppliers of energy metabolites to fuel the activity of hypothalamic POMC neurons that regulate energy homeostasis and as the sensors of the peripheral energy state upstream of these neurons. Further investigation into how tanycytes, which bridge the blood and CSF, perceive and translate this information for neurons could clear the way for potential treatments for metabolic disorders such as obesity and type 2 diabetes.

\section{Methods}

\section{Animals}

The experiments used WT C57Bl/6J and single-, double-, or triple-transgenic Cx43 $3^{\text {loxp/loxp }}$ (RRID_JAX008039), Pomc::Cre (RRID:IMSR_ JAX:005965), tdTomato ${ }^{\text {loxP-STOP-loxP }}$ (RRID:IMSR_JAX007914), and Cx $43^{\text {loxP/loxP }}$ tdTomato ${ }^{P O M C}$ mice maintained on a C57Bl6 background as detailed in the Supplemental Methods. All mice were bred and housed in a temperature- and humidity-controlled room on a 12-hour light/12hour dark cycle with ad libitum access to food and water.

\section{Stereotaxic delivery of viruses to manipulate the expression of Mct in POMC neurons and tanycytes}

In POMC neurons. AAV8[FLEXon]-SYN1 (EGFP:miR30_MCT2 [mSlc16a7] $)\left(1.17 \times 10^{13} \mathrm{PFU} / \mathrm{mL}\right.$, Vector Builder, Vector ID: VB200715-1173hzs) and AAV-GFP scrambled $\left(1.1 \times 10^{13} \mathrm{PFU} / \mathrm{mL}\right.$, VectorBuilder) viral vectors were injected into the ARH of POMC-Cre mice $(58,59)$. The ARH was targeted bilaterally, and adeno-associated viruses (AAVs) were delivered at a rate of $0.1 \mu \mathrm{L} / \mathrm{min}$ for 5 minutes ( $0.5 \mu \mathrm{L} /$ injection site) according to the following coordinates: $-1.5 \mathrm{~mm}$ posterior to bregma, $\pm 0.2 \mathrm{~mm}$ lateral to midline, and $-6 \mathrm{~mm}$ below the surface of the skull. Specific transduction of POMC neurons by AAVs was evaluated by immunohistochemistry. BW and food intake were recorded over 5 weeks after the surgery.

In tanycytes. WT $\mathrm{C} 57 \mathrm{Bl} / 6 \mathrm{~J}$ or tdTomato $^{\text {POMC }}$ mice were injected with either $2 \mu \mathrm{L}$ of a 1:1 mixture of AAV1/2[FLEXon]-CMV (EGFP:miR30_shRNA-MCT-1[mSlc16a1]_shRNA-MCT-4[mSlc16a3]) (at a titer of $4.4 \times 10^{9} \mathrm{gp} / \mu \mathrm{L}$; VectorBuilder, vector ID: VB2007161259yky) and AAV1/2-Dio2:Cre (at a titer of $\left.2.2 \times 10^{10} \mathrm{gp} / \mu \mathrm{L}\right)(27)(n=$ 4), or $2 \mu \mathrm{L}$ of a 1:1 mixture of AAV1/2[FLEXon]-CMV (EGFP:miR30_ scramble) (at a titer of $1.6 \times 10^{10} \mathrm{gp} / \mu \mathrm{L}$ ) and AAV1/2-Dio2:Cre (at a titer of $\left.2.2 \times 10^{10} \mathrm{gp} / \mu \mathrm{L}\right)(n=4)$ at a rate of $0.3 \mu \mathrm{L} / \mathrm{min}$. All animals underwent injection into the lateral ventricle (coordinates from bregma, anteroposterior: $-0.3 \mathrm{~mm}$; midline: $-1 \mathrm{~mm}$; and dorsoventral: -2.5 $\mathrm{mm}$ from the skull). 


\section{Stereotaxic TAT-Cre delivery}

Tanycyte-specific Cx43 deletion was performed using the Cre/LoxP system by stereotaxically infusing a TAT-Cre fusion protein into the $3 \mathrm{~V}$ $(2 \mu \mathrm{L} ; 0.3 \mu \mathrm{L} / \mathrm{min}$; anteroposterior/midline/dorsoventral coordinates: $-1.7 \mathrm{~mm} / 0 \mathrm{~mm} /-5.6 \mathrm{~mm}$ ) in isoflurane-anesthetized $C x 43^{\text {loxp/loxP }}$ mice or $C x 43^{\text {loxP/loxP }}$ tdTomato ${ }^{P O M C}$ mice, 1 week before experiments. The TATCre fusion protein was produced as described previously (4). Control mice were injected with a vehicle solution.

\section{Brain slice preparation}

Electrophysiological recordings were performed on living brain slices containing the tuberal region of the hypothalamus from 8- to 12-weekold mice as previously described (59). Briefly, mice were anesthetized with isoflurane and decapitated. The brain was removed and rapidly placed in ice-cold ACSF containing the following: $120 \mathrm{mM} \mathrm{NaCl}, 3.2$ $\mathrm{mM} \mathrm{KCl}, 1 \mathrm{mM} \mathrm{NaH}_{2} \mathrm{PO}_{4}, 26 \mathrm{mM} \mathrm{NaHCO}_{3}, 1 \mathrm{mM} \mathrm{MgCl}_{2}, 2 \mathrm{mM} \mathrm{CaCl}_{2}$, $2.5 \mathrm{mM}$ glucose (290 mOsm, pH 7.4) and bubbled with $95 \% \mathrm{O}_{2}$ to $5 \%$ $\mathrm{CO}_{2}$. Three $250 \mu \mathrm{m}$ thick coronal hypothalamic slices containing the arcuate nucleus were cut using a VT1200 vibratome (Leica). The slices were incubated at $34^{\circ} \mathrm{C}$ in oxygenated ACSF for a recovery period of 1 hour, and then at room temperature until the recording.

\section{Patch-clamp recording}

For patch-clamp recording, individual brain slices were placed in a submerged recording chamber (Warner Instruments), immobilized by a nylon grid, and continuously perfused at a rate of $3 \mathrm{~mL} / \mathrm{min}$ with oxygenated ACSF maintained at $32.8^{\circ} \mathrm{C}$ by a heater controller (TC-344C, Warner Instruments). POMC neurons and ARH tanycytes were visualized and identified under $\times 40$ magnification using an upright fluorescence microscope with infrared differential interference contrast (DM-LFSA, Leica) and an ORCA-Flash4.0 digital CMOS camera (Hamamatsu). Recording pipettes were pulled from borosilicate glass capillaries $(1.5 \mathrm{~mm}$ outer diameter, $1.12 \mathrm{~mm}$ inner diameter; World Precision Instruments) using a P1000 Flaming Brown puller (Sutter Instruments) and had a resistance of 4-6 M $\Omega$ when filled with an internal solution containing the following:

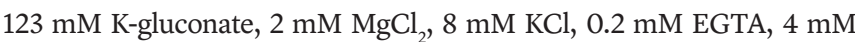
$\mathrm{Na}_{2}$-ATP, $0.3 \mathrm{mM} \mathrm{Na}$-GTP, and $10 \mathrm{mM}$ HEPES, pH 7.3, with KOH. In paired recordings of a tanycyte and a neuron, sodium L-lactate $(5 \mathrm{mM}$, Alfa Aesar, catalog L14500, Chemical Abstracts Service [CAS]: 867-56-1) was also added to the internal solution of the tanycyte pipette. Pipettes were placed in contact with a POMC neuron or an ARH tanycyte using a PCS-5400 micromanipulator (Thorlabs). Whole-cell patch-clamp recordings were performed in current-clamp mode using a Multiclamp 700B Amplifier, digitized with the Digidata 1322A interface and acquired with pClamp 10.2 software (Molecular Devices). Data were filtered at 1 $\mathrm{kHz}$ and sampled at $5 \mathrm{kHz}$.

For exogenous glucose deprivation studies, the osmolarity of the ACSF containing $0 \mathrm{mM}$ D-glucose was adjusted with sucrose. All drugs tested (4-CIN, $500 \mu \mathrm{M}$, MilliporeSigma, catalog C2020, CAS: 2816641-8; sodium oxamate, $10 \mathrm{mM}$, MilliporeSigma, catalog O2751, CAS: 565-73-1; sodium L-lactate, $5 \mathrm{mM}$, Alfa Aesar; sodium pyruvate, $5 \mathrm{mM}$, MilliporeSigma, catalog P2256, CAS: 113-24-6; and CBX, $50 \mu \mathrm{M}$, MilliporeSigma, catalog C4790, CAS: 7421-40-1) were applied to the perfusion system after stable baseline recording.

Recordings were analyzed using Clampfit 10.2 in pClamp software (Molecular Devices). For each recording, the membrane potential and mean firing rate were determined before and during the bath application of drugs. Neurons were considered responsive when a change of more than $20 \%$ in the firing rate was observed. The peak response was determined, and the number of spikes was counted 3 minutes before and after the peak effect.

For glucose and dye diffusion experiments, the internal solution contained the fluorescent glucose derivate 2-NBDG $(2 \mathrm{mg} / \mathrm{mL}$, Invitrogen, Thermo Fisher Scientific, catalog N13195, CAS: 1 86689-07-6) or biocytin (2 mg/mL, MilliporeSigma, catalog B4261, CAS: 576-19-2), respectively. Recorded cells were loaded with these solutions for 20 minutes in current-clamp mode. The intercellular diffusion of 2-NBDG was captured online with a digital camera just after recording, and the diffusion distance was analyzed with Fiji software. Biocytin diffusion was revealed by Alexa Fluor 488 streptavidin (Invitrogen, Thermo Fisher Scientific, catalog S11223) as detailed in the Supplemental Methods and analyzed using an Axio Imager Z2 Apotome microscope (AxioCam 504 MRm camera, Zeiss). To inhibit gap-junctional communication, slices were pretreated for 20 minutes with $50 \mu \mathrm{M} \mathrm{CBX}$.

For dye diffusion during paired tanycyte-neuron recordings, 1 ARH tanycyte was recorded and filled with the gap junction-permeable lucifer yellow $\mathrm{CH}$ dilithium salt $(1 \mathrm{mg} / \mathrm{mL}$; MilliporeSigma, catalog L0259, CAS: 67769-47-5) through the patch pipettes; 1 neighboring POMC neuron was then similarly recorded and filled with the membrane-impermeable dye Alexa Fluor 594 Hydrazide $(40$ mM; Invitrogen, Thermo Fisher Scientific, catalog A10438). Images were captured 20 minutes after recording using a digital camera.

\section{Primary cultures of tanycytes}

Primary cultures of tanycytes were generated by isolating the ME of 10-day-old Sprague-Dawley rats (Janvier Laboratories), as described previously (26), and processed as described in the Supplemental Methods.

\section{FACS and $q P C R$}

FACS isolation of hypothalamic neurons and tanycytes and subsequent GPCR analyses were performed according to previously described protocols $(33,60)$, with some adaptations, as detailed in the Supplemental Methods.

\section{Immunochemistry}

Immunocytofluorescence protocols are detailed in the Supplemental Methods. The following primary antibodies were used: anti-Cx43 (MilliporeSigma, catalog AB1727, RRID:AB_2294609, 1:200); anti-MCT1 (MilliporeSigma, catalog AB1286, RRID:AB_11212410, 1:100); antiMCT4 (MilliporeSigma, 1:200); anti-vimentin (MilliporeSigma, cata$\log$ AB3314P, RRID:AB_2286063, 1:1000 and Dako, catalog M0725, RRID:AB_10013485, 1:100); anti-NeuN (1:1000; MilliporeSigma, catalog MAB377, RRID:AB_2298772); anti-POMC propeptide (1:200; Phoenix Pharmaceuticals, catalog H02930, RRID:AB_2307442); and anti-GFP (1:1000; Invitrogen, Thermo Fisher Scientific, catalog A10262, RRID:AB_2534023). Immunofluorescence images were captured using a Zeiss Axio Imager Z2 Apotome microscope or a Zeiss LSM 710 confocal microscope.

\section{Metabolic studies}

Analysis of basal metabolism. Mice were analyzed for total energy expenditure, $\mathrm{O}_{2}$ consumption, $\mathrm{CO}_{2}$ production, food intake, and ambulatory movements (total beam breaks/hour) using calorimetric cages (TSE Systems) and standard procedures published by others (61, 
62). Mice were individually housed and acclimatized to the cages for 48 hours before experimental measurements.

$B W$ and composition. Fat and lean tissue mass was determined by nuclear magnetic resonance (MiniSpec mq 7.5, RMN Analyser, Bruker) according to the manufacturer's recommendations.

\section{In vivo brain microdialysis}

Male $C x 43^{\text {loxP/loxP }}$ and $C x 43^{\text {TanycyteкO }}$ mice weighing $30-35$ g were deeply anesthetized with isoflurane ( $3 \%$ in $1 \mathrm{~L} / \mathrm{min}$ airflow) in an induction chamber and placed in a stereotaxic apparatus equipped with a mask to maintain anesthesia throughout the experiment (isoflurane $1 \%$ in $0.7 \mathrm{~L} / \mathrm{min}$ airflow). Core body temperature was maintained at $37^{\circ} \mathrm{C}$ with an electrical blanket controlled by a thermostat. A microdialysis cannula (CMA7, $6 \mathrm{kDa}, 2 \mathrm{~mm}$ membrane length; CMA Microdialysis $\mathrm{AB}$ ) was implanted over the hypothalamic area using stereotaxic coordinates (anteroposterior: -1.4 , lateral: -0.3 , ventral: $-6.0 \mathrm{~mm}$ relative to bregma). The microdialysis probe was then perfused with sterile ACSF (CMA Perfusion Fluid CNS containing $147 \mathrm{mM} \mathrm{NaCl}, 2.7 \mathrm{mM} \mathrm{KCl}, 1.2 \mathrm{mM} \mathrm{CaCl}_{2}$, and $0.85 \mathrm{mM} \mathrm{MgCl}_{2}$ ) at a rate of $2 \mu \mathrm{L} / \mathrm{min}$ using a microinjection pump (CMA 402; CMA). Following a stabilization period of 60 minutes (2 dialysates of 10 minutes each were collected at 40 minutes and 50 minutes), a sterile vehicle injection (i.p., $0.9 \% \mathrm{NaCl}$ ) was administered to the mice, and 4 dialysates of 10 minutes each were recovered. After $100 \mathrm{~min}$ utes, a glucose solution was administered to the mice (i.p., $2 \mathrm{~g} / \mathrm{kg}$ in saline), and 7 dialysates of 10 minutes each were recovered. Brain dialysates were placed in a fraction collector (CMA/820) during the experiment and immediately stored at $-80^{\circ} \mathrm{C}$ until analysis. At the end of the experiment, mice were euthanized by decapitation, and brains were stored immediately in fresh $4 \%$ paraformaldehyde. The lactate content of microdialysates from the brain was measured using the luminometric Lactate-Glo Assay Kit (Promega), according to the manufacturer's protocol, and a Varioskan LUX microplate reader (Thermo Fisher Scientific).

\section{Statistics}

Data are presented as the mean \pm SEM. All statistical analyses were performed using GraphPad Prism, version 7 (GraphPad Software) and are reported in Supplemental Table 1. To compare 2 groups, an unpaired or paired 2-tailed Student's $t$ test was used for normal distri- butions and a Wilcoxon matched-pairs test or a Mann-Whitney $U$ test was performed for non-Gaussian distributions. To compare several groups, a 1-way ANOVA followed by Tukey's post hoc multiple comparison test or a 2-way ANOVA followed by an uncorrected Fisher's least significant difference (LSD) test was used. A Repeated-measures ANOVA was used when multiple measurements were made over time in the same groups. $P$ values of less than 0.05 were considered statistically significant.

\section{Study approval}

Ethics approval for animal studies was obtained from the French Ministry of National Education, Higher Education and Research (APAFIS no. 2617-2015110517317420 v5 and APAFIS no. 133872017122712209790 v9) and by the Ethics Committee of the University of Santiago de Compostela, in accordance with European Union norms for experimental animals.

\section{Author contributions}

TL and JC performed the electrophysiological and neuroanatomical experiments. FS performed the experiments in primary cultures. JC, EC, and MI conducted the metabolic phenotyping of the mice. DF, MI, and IMC performed the microdialysis experiments. TL, MI, DF, NDSL, VH, and HMF conducted experiments involving AAVs. TL, JC, MI, DF, SR, MS, RN, and VP designed and planned the study. All authors contributed to the preparation of the manuscript.

\section{Acknowledgments}

This work was supported by the European Research Council (ERC) Synergy Grant WATCH (grant 810331, to RN, VP, and MS), the H2020-MSCA-IF-2014 (grant ID656657, to JC), and the H2020-MSCA-IF-2016 (grant 748134, to MI), and the Region Hauts-de-France VisionAIRR (grant 189090). We thank Meryem Tardivel (confocal microscopy) and Nathalie Jouy (flow cytometry) from the BioImaging Center of Lille (BiCeL) and Julien Devassine (animal core facility) of the UMS2014-US41 for their expert technical support.

Address correspondence to: Vincent Prevot, INSERM U1172, Bâtiment Biserte, Place de Verdun, 59045 Lille Cedex, France. Phone: 33.320.62.20.64; Email: vincent.prevot@inserm.fr.
1. De Vivo DC, et al. Defective glucose transport across the blood-brain barrier as a cause of persistent hypoglycorrhachia, seizures, and developmental delay. NEngl JMed.1991;325(10):703-739.

2. Wang D, et al. A mouse model for Glut-1 haploinsufficiency. Hum Mol Genet. 2006;15(7):1169-1179.

3. Fox PT, et al. Nonoxidative glucose consumption during focal physiologic neural activity. Science. 1988;241(4864):462-464.

4. Langlet F, et al. Tanycytic VEGF-A boosts blood-hypothalamus barrier plasticity and access of metabolic signals to the arcuate nucleus in response to fasting. Cell Metab. 2013;17(4):607-617.

5. Dunn-Meynell AA, et al. Relationship among brain and blood glucose levels and spontaneous and glucoprivic feeding. J Neurosci. 2009;29(21):7015-7022.

6. Claret M, et al. AMPK is essential for energy homeostasis regulation and glucose sensing by POMC and AgRP neurons. JClin Invest. 2007;117(8):2325-2336.

7. Parton LE, et al. Glucose sensing by POMC neurons regulates glucose homeostasis and is impaired in obesity. Nature. 2007;449(7159):228-232.

8. Levin BE, et al. Neuronal glucosensing: what do we know after 50 years? Diabetes. 2004;53(10):2521-2528.

9. Fioramonti X, et al. Characterization of glucosensing neuron subpopulations in the arcuate nucleus: integration in neuropeptide $\mathrm{Y}$ and pro-opio melanocortin networks? Diabetes. 2007;56(5):1219-1227.

10. Levin BE. Neuronal glucose sensing: still a physiological orphan? Cell Metab. 2007;6(4):252-254.

11. Fishman RA. Carrier transport of glucose between blood and cerebrospinal fluid. Am J
Physiol. 1964;206:836-844.

12. Nigrovic LE, et al. Relationship between cerebrospinal fluid glucose and serum glucose. $N$ Engl J Med. 2012;366(6):576-578.

13. Magistretti PJ, Allaman I. Lactate in the brain: from metabolic end-product to signalling molecule. Nat Rev Neurosci. 2018;19(4):235-249.

14. Prevot V, et al. The versatile tanycyte: a hypothalamic integrator of reproduction and energy metabolism. Endocr Rev. 2018;39(3):333-368.

15. Garcia-Caceres C, et al. Role of astrocytes, microglia, and tanycytes in brain control of systemic metabolism. Nat Neurosci. 2019;22(1):7-14.

16. Parsons MP, Hirasawa M. ATP-sensitive potassium channel-mediated lactate effect on orexin neurons: implications for brain energetics during arousal. J Neurosci. 2010;30(24):8061-8070.

17. Lam TK, et al. Regulation of blood glucose by 
hypothalamic pyruvate metabolism. Science. 2005;309(5736):943-947.

18. Sanders NM, et al. Third ventricular alloxan reversibly impairs glucose counterregulatory responses. Diabetes. 2004;53(5):1230-1236.

19. Elizondo-Vega R, et al. The role of tanycytes in hypothalamic glucosensing. J Cell Mol Med. 2015;19(7):1471-1482.

20. Frayling C, et al. ATP-mediated glucosensing by hypothalamic tanycytes. J Physiol. 2011;589(Pt 9):2275-2286.

21. Cortes-Campos C, et al. MCT expression and lactate influx/efflux in tanycytes involved in glia-neuron metabolic interaction. PLoS One. 2011;6(1):e16411.

22. Nilaweera K, et al. Photoperiodic regulation of glycogen metabolism, glycolysis, and glutamine synthesis in tanycytes of the Siberian hamster suggests novel roles of tanycytes in hypothalamic function. Glia. 2011;59(11):1695-1705.

23. Pellerin L, Magistretti PJ. Glutamate uptake into astrocytes stimulates aerobic glycolysis: a mechanism coupling neuronal activity to glucose utilization. Proc Natl Acad Sci U S A. 1994;91(22):10625-10629.

24. Clasadonte J, et al. Connexin 43-mediated astroglial metabolic networks contribute to the regulation of the sleep-wake cycle. Neuron. 2017;95(6):1365-1380

25. Balland E, et al. Hypothalamic tanycytes are an ERK-gated conduit for leptin into the brain. Cell Metab. 2014;19(2):293-301.

26. Prevot $\mathrm{V}$, et al. Activation of erbB-1 signaling in tanycytes of the median eminence stimulates transforming growth factor beta1 release via prostaglandin E2 production and induces cell plasticity. J Neurosci. 2003;23(33):10622-10632.

27. Muller-Fielitz H, et al. Tanycytes control the hormonal output of the hypothalamic-pituitary-thyroid axis. Nat Commun. 2017;8(1):484.

28. Rouach N, et al. Astroglial metabolic networks sustain hippocampal synaptic transmission. Science. 2008;322(5907):1551-1555.

29. Szilvasy-Szabo A, et al. Localization of connexin 43 gap junctions and hemichannels in tanycytes of adult mice. Brain Res. 2017;1673:64-71.

30. Recabal A, et al. Connexin-43 gap junctions are responsible for the hypothalamic tanycyte-coupled network. Front Cell Neurosci. 2018;12:406.

31. Martinez F, et al. The median eminence as the hypothalamic area involved in rapid transfer of glucose to the brain: functional and cellular mechanisms. JMol Med (Berl). 2019;97(8):1085-1097.
32. Parkash J, et al. Semaphorin7A regulates neuroglial plasticity in the adult hypothalamic median eminence. Nat Commun. 2015;6:6385.

33. Duquenne $M$, et al. Leptin brain entry via a tanycytic LepR:EGFR shuttle controls lipid metabolism and pancreas function. Nat Metab. 2021;8(8):13-22.

34. Steffens AB, et al. Penetration of peripheral glucose and insulin into cerebrospinal fluid in rats. Am J Physiol. 1988;255(2 Pt 2):R200-204.

35. Lam CK, et al. Central lactate metabolism regulates food intake. Am JPhysiol Endocrinol Metab. 2008;295(2):E491-E496.

36. Cha SH, Lane MD. Central lactate metabolism suppresses food intake via the hypothalamic AMP kinase/malonyl-CoA signaling pathway. Biochem Biophys Res Commun. 2009;386(1):212-216.

37. Lee YS, et al. A POMC variant implicates beta-melanocyte-stimulating hormone in the control of human energy balance. Cell Metab. 2006;3(2):135-140.

38. Denis RG, et al. Central orchestration of peripheral nutrient partitioning and substrate utilization: implications for the metabolic syndrome. Diabetes Metab. 2014;40(3):191-197.

39. Krashes MJ, et al. Melanocortin-4 receptor-regulated energy homeostasis. Nat Neurosci. 2016;19(2):206-219.

40. Gautron L, et al. Neural control of energy balance: translating circuits to therapies. Cell. 2015;161(1):133-145.

41. Lelliott C, Vidal-Puig AJ. Lipotoxicity, an imbalance between lipogenesis de novo and fatty acid oxidation. Int JObes Relat Metab Disord. 2004;28(Suppl 4):S22-S28.

42. Mayer J. Glucostatic mechanism of regulation of food intake. NEngl JMed. 1953;249(1):13-16.

43. Oomura Y, et al. Reciprocal activities of the ventromedial and lateral hypothalamic areas of cats. Science. 1964;143(3605):484-485.

44. Machler P, et al. In vivo evidence for a lactate gradient from astrocytes to neurons. Cell Metab. 2016;23(1):94-102.

45. Leen WG, et al. Cerebrospinal fluid analysis in the workup of GLUT1 deficiency syndrome: a systematic review. JAMA Neurol. 2013;70(11):1440-1444.

46. Garcia-Caceres C, et al. Astrocytic insulin signaling couples brain glucose uptake with nutrient availability. Cell. 2016;166(4):867-880.

47. Gruber T, et al. Obesity-associated hyperleptinemia alters the gliovascular interface of the hypothalamus to promote hypertension. Cell Metab. 2021;33(6):1155-1170.
48. Guillebaud F, et al. Glial endozepines reverse high-fat diet-induced obesity by enhancing hypothalamic response to peripheral leptin. $\mathrm{Mol}$ Neurobiol. 2020;57(8):3307-3333.

49. Nuzzaci D, et al. Postprandial hyperglycemia stimulates neuroglial plasticity in hypothalamic POMC neurons after a balanced meal. Cell Rep. 2020;30(9):3067-3078.

50. Guillebaud F, et al. Blockade of glial connexin 43 hemichannels reduces food intake. Cells. 2020;9(11):2387.

51. Kotini M, et al. Gap junction protein Connexin- 43 is a direct transcriptional regulator of $\mathrm{N}$-cadherin in vivo. Nat Commun. 2018;9(1):3846.

52. Yaswen L, et al. Obesity in the mouse model of pro-opiomelanocortin deficiency responds to peripheral melanocortin. Nat Med. 1999;5(9):1066-1070.

53. Challis BG, et al. Mice lacking pro-opiomelanocortin are sensitive to high-fat feeding but respond normally to the acute anorectic effects of peptide-YY(3-36). Proc Natl Acad Sci U S A. 2004;101(13):4695-4700.

54. Huszar D, et al. Targeted disruption of the melanocortin- 4 receptor results in obesity in mice. Cell. 1997;88(1):131-141.

55. Zheng $\mathrm{H}$, et al. Brain stem melanocortinergic modulation of meal size and identification of hypothalamic POMC projections. Am J Physiol Regul Integr Comp Physiol. 2005;289(1):R247-R258.

56. Zhan C, et al. Acute and long-term suppression of feeding behavior by POMC neurons in the brainstem and hypothalamus, respectively. J Neurosi. 2013;33(8):3624-3632.

57. Cortes-Campos $\mathrm{C}$, et al. MCT2 expression and lactate influx in anorexigenic and orexigenic neurons of the arcuate nucleus. PLoS One. 2013;8(4):e62532.

58. Quinones M, et al. Sirt3 in POMC neurons controls energy balance in a sex- and diet-dependent manner. Redox Biol. 2021;41:101945.

59. Al-Massadi O, et al. MCH regulates SIRT1/FoxO1 and reduces POMC neuronal activity to induce hyperphagia, adiposity, and glucose intolerance. Diabetes. 2019;68(12):2210-2222.

60. Messina A, et al. A microRNA switch regulates the rise in hypothalamic $\mathrm{GnRH}$ production before puberty. Nat Neurosci. 2016;19(6):835-844.

61. Tschop MH, et al. A guide to analysis of mouse energy metabolism. Nat Methods. 2012;9(1):57-63.

62. Denis RG, et al. Palatability can drive feeding independent of AgRP neurons. Cell Metab. 2015;22(4):646-657. 